\title{
Leading Edge Noise Predictions Using Anisotropic Synthetic Turbulence
}

\author{
Fernando Gea-Aguilera*, James Gill† Xin Zhang; \\ Xiaoxian Chen, ${ }^{\dagger}$ and Thomas Nodé-Langlois ${ }^{\S}$ \\ ${ }^{*} \dagger$ Faculty of Engineering and the Environment, \\ University of Southampton, Southampton, SO16 7QF, UK. \\ ¥The Hong Kong University of Science and Technology, \\ Clear Water Bay, Kowloon, Hong Kong SAR, China. \\ $\S$ Acoustics - Numerical Methods, \\ Airbus Operations S.A.S., 31060 Toulouse, France.
}

\begin{abstract}
An advanced digital filter method is presented to generate divergence-free synthetic turbulence with homogeneous anisotropic velocity spectra. The resulting fluctuating velocity field is obtained through a superposition of anisotropic Gaussian eddies. This method is used to generate a two-dimensional turbulent flow with the key statistics of homogeneous axisymmetric turbulence. This type of turbulence has been reported in aero-engine intakes, fan wakes and open-jet wind tunnel experiments. The advanced digital filter method is implemented in a linearized Euler solver in order to investigate potential effects of anisotropic turbulence on leading edge noise. Computational aeroacoustic simulations are performed for anisotropic turbulence with streamwise-to-transverse length scale ratios ranging from 0.33 to 3 on a number of isolated airfoil configurations, including variations in mean flow Mach number, airfoil thickness and angle of attack. Noise reduction due to airfoil thickness is assessed on a NACA 0012 airfoil at zero angle of attack, showing similar trends for both isotropic and moderately anisotropic turbulent flows. Effects of anisotropic turbulence on noise become evident for airfoil configurations at non-zero angle of attack.
\end{abstract}

Keywords: computational aeroacoustics, leading edge noise, anisotropic synthetic turbulence, digital filter

\section{Introduction}

$\mathrm{E}_{\mathrm{com}}^{\mathrm{N}}$ NGINES, high-lift devices and landing gears are the main contributors to broadband noise generated by

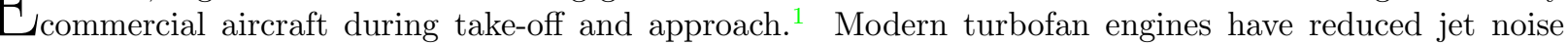
compared to older designs by increasing the bypass ratio. The main source of engine broadband noise is now associated with the interaction of the fan wakes with the Outlet Guide Vanes (OGVs). ${ }^{2}$ Leading edge noise, also known as turbulence-airfoil interaction noise, is the underlying cause of the fan wake-OGV interaction noise, as well as the turbulence ingestion noise, including the interaction between the inlet boundary layer and the fan. Additionally, turbulence-airfoil interaction noise is also relevant in Contra-Rotating Open-Rotors (CRORs), helicopter rotors and wind turbines.

In order to obtain a better understanding of leading edge noise, a number of analytical,,$^{3,4}$ experimental ${ }^{5-7}$ and numerical ${ }^{8-12}$ studies have examined the interaction of isolated airfoils with homogeneous and isotropic

*PhD Student, Airbus Noise Technology Centre, AIAA Student Member, F.Gea-Aguilera@soton.ac.uk.

${ }^{\dagger}$ Research Fellow, Airbus Noise Technology Centre.

¥Swire Professor of Aerospace Engineering, Department of Mechanical and Aerospace Engineering, Associate Fellow, AIAA, aexzhang@ust.hk.

$\S$ Acoustic Engineer, Acoustics Department, Airbus. 
turbulence. However, the majority of analytical models are restricted to flat plate theory and do not account for real geometry airfoils. Experiments using grid-generated turbulence produce a turbulent inflow that presents a certain degree of anisotropy. Furthermore, the effect of the angle of attack on the leading edge noise is difficult to quantify in open-jet wind tunnel facilities, where the jet deflection changes the effective angle of attack that is perceived by the airfoil. Consequently, low-cost numerical simulations using Computational AeroAcoustics (CAA) solvers have been used to study complex isolated airfoil configurations. These usually solve the full or linearized Euler equations using isotropic synthetic turbulence.

To date, variations in leading edge noise due to isotropic turbulence interacting with real geometry airfoils are well-understood. An increase in airfoil thickness produces a significant reduction on the noise levels at high frequencies, ${ }^{5,10}$ whereas changes in angle of attack and camber produce minor effects on noise levels. ${ }^{6,7,11}$ However, few works have addressed the effects of anisotropic turbulence on leading edge noise, despite the anisotropic features of turbulence in the engine intake ${ }^{13-16}$ and fan wakes. ${ }^{17}$ Figure 1 shows a schematic representation of anisotropic turbulence in turbofan engines.

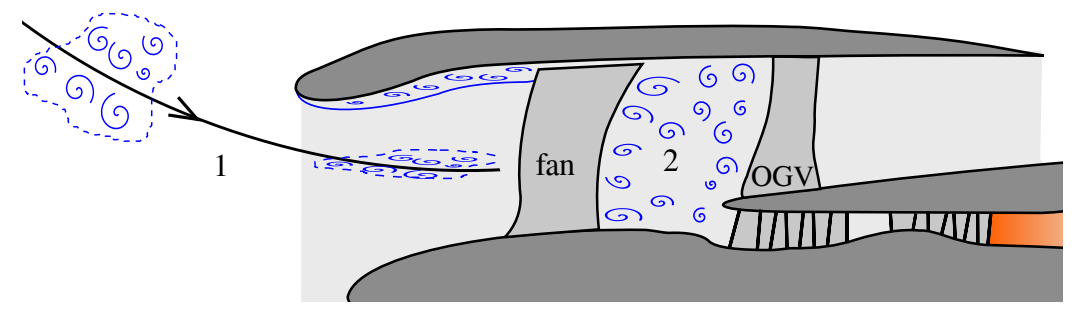

Figure 1. Anisotropic turbulence in a turbofan engine. (1) Turbulence ingestion and (2) fan wakes.

Turbulence length scales of the order of $10^{2}$ times larger in the axial direction when compared to lateral direction were reported in static test conditions of a fan intake. ${ }^{13}$ Although this anisotropy is significantly reduced in flight conditions, ${ }^{14}$ boundary layer turbulence, which interacts with the fan blade tip, also presents strong anisotropy. Highly anisotropic turbulent flows at the fan intake could be modeled by assuming homogeneous axisymmetric turbulence. ${ }^{15,16}$ This type of turbulence corresponds to a simple modeling of an anisotropic flow, in which the root-mean-square velocity component and length scale vary in a prevailing axis. Additionally, axisymmetric turbulence has also been reported in experimental measurements of fan wakes, ${ }^{17}$ where the ratio of streamwise-to-transverse integral length scales ranges from 1.4 to 4 , depending on the radial location. It should be noted that for homogeneous and isotropic turbulence, the streamwiseto-transverse integral length scale ratio is $2 .{ }^{18}$

Noise measurements of turbulence-airfoil interaction in open-jet wind tunnels are often affected by a certain degree of anisotropy. ${ }^{5,19}$ For instance, Paterson and Amiet ${ }^{5}$ reported a streamwise-to-transverse integral length scale ratio of 1.25. Current analytical models that are used to predict turbulence ingestion noise and fan-OGV interaction noise are highly sensitive to input turbulence spectra. ${ }^{16,20}$ Thus, a good understanding of the effects of anisotropic turbulence on isolated airfoil configurations can help to improve noise predictions.

The aim of the current work is twofold. Firstly, an advanced digital filter method to produce anisotropic synthetic turbulence is presented and validated. The method, which is an extension of a previous work on isotropic turbulence, ${ }^{12}$ is able to produce divergence-free homogeneous anisotropic turbulence with the desired spectral content. Secondly, the effects of anisotropic turbulence on leading edge noise predictions are studied under the assumption of homogeneous axisymmetric turbulence. To this end, a number of isolated airfoil configurations are investigated for different degrees of anisotropy in the turbulent inflow. The study also addresses the effects of the freestream Mach number, airfoil thickness and angle of attack.

\section{Previous Work}

A bibliographic review of the methods to generate anisotropic synthetic turbulence is presented in Section II.A. The methods presented herein have been conceived for either CAA or Large Eddy Simulations (LES). The characteristics of some methods developed for LES, such as divergence-free fluctuating velocity, makes them suitable for CAA simulations. Then, studies on leading edge noise using anisotropic turbulence are presented in Section II.B. The latter includes works on isolated airfoil configurations, as well as airfoil cascades. Preliminary findings of the effects of anisotropic turbulence on leading edge noise are highlighted. 


\section{II.A. Studies to Generate Anisotropic Synthetic Turbulence}

Synthetic turbulence methods are typically based on a summation of Fourier modes, digital filters or synthetic eddies. These methods define velocity fluctuations that fulfill the divergence-free condition, which is required to avoid the introduction of spurious noise in CAA simulations.

An early work by Kraichnan ${ }^{21}$ proposed to generate stochastic velocity fields by superposing a number of Fourier modes. The method, which was conceived for Gaussian spectra, was able to produce isotropic turbulence. Based on Kraichnan's work, ${ }^{21}$ Smirnov et al. ${ }^{22}$ generated anisotropic turbulence through a scaling and orthogonal transformation of the velocity correlation tensor. This technique, which was developed for LES applications, produces a fluctuating velocity field that is not completely divergence-free in cases that consider highly anisotropic turbulence. In order to account for non-Gaussian energy spectra, Huang et al. ${ }^{23}$ proposed to generate a number of independent velocity fields following the approach of Smirnov et al. ${ }^{22}$ The superposition of all the flow fields matches a desired turbulence spectrum. Recently, Castro et al. ${ }^{24}$ introduced a number of parameters to better control the statistical properties of the anisotropic velocity generated with Huang et al.'s method. ${ }^{23}$ Further developments were performed by Batten et al. ${ }^{25}$ who proposed a Cholesky decomposition of the Reynolds stress tensor to characterize the amplitude of Fourier modes. Billson et al. ${ }^{26}$ adapted Smirnov et al.'s transformation ${ }^{22}$ to include anisotropy in the Reynolds stress tensor and length scales when using a Stochastic Noise Generation and Radiation (SNGR) based method. ${ }^{27,28}$ Recently, Yu and $\mathrm{Bai}^{29}$ have proposed the use of a potential vector in Fourier mode methods. Then, the fluctuating velocity is obtained by taking the curl of the vector potential, which ensures the divergence-free condition.

In the area of digital filter methods for LES, Klein et al. ${ }^{30}$ developed a method to match anisotropic velocity correlations by filtering random data with filters of different lengths in each spatial direction. Nevertheless, the method was not divergence-free. Based on Klein et al.'s work, ${ }^{30}$ Ewert $^{31}$ developed the Random Particle-Mesh method to generate synthetic turbulence for CAA applications. This method could deal with anisotropic flows by using Smirnov et al.'s transformation. ${ }^{22}$ Siefert and Ewert ${ }^{32}$ introduced a hierarchy of filters to account for anisotropy in the Reynolds stress tensor and length scales.

Synthetic eddy methods can also deal with anisotropic turbulence by using eddies with different amplitude and length scales in each spatial direction. ${ }^{33,34}$ For instance, Poletto et al. ${ }^{33}$ defined the eddies in the local principal axes, where the Reynolds stress tensor is diagonal. Then, a rotation matrix was applied to define the eddies in the global reference system, which causes the eddies to be oriented at different inclinations.

\section{II.B. Studies of Leading Edge Noise using Anisotropic Turbulence}

Relatively few experimental works have investigated the effects of anisotropic turbulence on leading edge noise. Unlike classic studies using nearly isotropic grid-generated turbulence, ${ }^{5,6,7}$ Olsen and Wagner ${ }^{35}$ tested a number of symmetric airfoils at zero angle of attack interacting with a jet flow. The airfoils were placed within the shear layer of the jet, where anisotropic turbulence occurs. The study showed a linear noise reduction when comparing sound pressure level from thick and thin airfoils. Hall et al. ${ }^{36}$ measured the noise from thick airfoils interacting with anisotropic turbulence from a boundary layer. Variations in the leading edge radius affected measured noise levels and led to local changes of airfoil angle of attack.

A study on the effects of anisotropic turbulence on leading edge noise was performed by Devenport et $a l .{ }^{7}$ Noise predictions on a NACA 0015 airfoil were performed by means of a panel method ${ }^{37}$ and the axisymmetric turbulence spectra proposed by Kerschen and Gliebe. ${ }^{15}$ The latter, which is based on a previous work by Chandrasekhar, ${ }^{38}$ provides a spectral representation of the turbulent flow by assuming homogeneous turbulence. Devenport et al. ${ }^{7}$ showed a significant increase in noise levels with increasing angle of attack, when the streamwise length scale was twice the transverse length scale. However, the study was limited to frequencies below $1000 \mathrm{~Hz}$ at a freestream Mach number of 0.08 . It should be noted that the effect of angle of attack on noise was found to be negligible for airfoils in isotropic turbulence. ${ }^{6,7,11}$

The homogeneous axisymmetric turbulence model of Kerschen and Gliebe ${ }^{15}$ has also been used by Hanson $^{39}$ and Posson et al. ${ }^{20}$ in flat plate cascade models. In particular, Posson et al. ${ }^{20}$ used an streamwiseto-transverse length scale ratio of 2 . This length scale ratio shifted the maximum spectrum level of noise towards lower frequencies and reduced the noise level up to $16 \mathrm{~dB}$ at high frequencies, when compared to the noise prediction using isotropic turbulence.

A different axisymmetric turbulence model, based on rapid distortion theory, ${ }^{40}$ was used by Atassi et al. ${ }^{16}$ when studying turbulence ingestion noise. For turbulence stretched in the streamwise direction, the peak 
noise level moved to lower frequencies. A decrease in noise levels was also reported in mid-range frequencies.

In the area of CAA simulations, Gill et al. ${ }^{11}$ studied the airfoil response to constant amplitude gusts on thick airfoils at non-zero angle of attack. It was shown that the peak noise level was shifted to nonzero transverse wavenumbers and increased in amplitude at moderate angles of attacks. This suggests that anisotropic turbulence could play a significant role in the leading edge noise of airfoils at non-zero angle of attack.

\section{CAA method}

The present work uses a well-established high-order finite difference numerical solver that has been used in prior CAA studies. ${ }^{41-43}$ In the current investigation, the LEEs are solved in the time-domain by using a 4th-order implicit spatial scheme. ${ }^{44}$ The numerical solution is filtered after each time step for numerical stability. ${ }^{45}$ The time derivatives are obtained by using a 4-6 Low Dispersion and Dissipation Runge-Kutta (LDDRK) scheme. ${ }^{46}$

A slip-wall boundary condition is used on the airfoil surface. Additionally, implicit buffer zones ${ }^{47}$ are applied in order to prevent sound waves from being reflected at the edge of the CAA domain.

Numerical simulations presented in this work were run with mean flow density $\rho_{0}=1.2 \mathrm{Kg} / \mathrm{m}^{3}$ and speed of sound $c_{0}=340 \mathrm{~m} / \mathrm{s}$. Turbulence velocity spectra are normalized by using $L_{r e f}=1 \mathrm{~m}$ and $U_{r e f}=1 \mathrm{~m} / \mathrm{s}$. The reference values for the Sound Pressure Level (SPL) and sound PoWer Level (PWL) calculations are $2 \times 10^{-5} \mathrm{~N} / \mathrm{m}^{2}$ and $1 \times 10^{-12} \mathrm{~W}$, respectively.

All computations used the IRIDIS 4 high performance computing facility at the University of Southampton.

\section{Homogeneous Anisotropic Synthetic Turbulence}

The majority of methods to generate anisotropic synthetic turbulence focus on reproducing the Reynolds stress tensor, which is related to one-point velocity correlations. However, the spectral content of the turbulent flow plays an important role in broadband noise predictions of turbulence-airfoil interaction. Turbulent velocity spectra are obtained through Fourier transform of the two-point velocity correlation tensor. ${ }^{18}$ The assumption of spatially homogeneous turbulence results in closed-form expressions for the velocity spectra, which is convenient for leading edge noise predictions.

Synthetic eddy methods usually require a large number of constraints and input parameters to obtain the correct statistics of a turbulent flow. For instance, Kim and Haeri ${ }^{48}$ used 15 constraints to reproduce the isotropic von Kármán spectrum in three-dimensional simulations. Fourier mode methods struggle to produce anisotropic turbulent flows that are completely divergence-free. Moreover, a large number of modes is require to obtain a turbulent flow with well-defined velocity spectra, which increases the computational cost.

In this work, further developments of an advanced digital filter method ${ }^{12}$ are presented to generate spatially homogeneous, but anisotropic, synthetic turbulence. In Section IV.A, the equations of two-dimensional Gaussian eddies are reviewed for isotropic turbulence and extended for anisotropic turbulence. Input parameters to introduce Gaussian eddies through an inlet section are also given. The resulting turbulent flow is homogeneous, with well-defined Gaussian velocity spectra, and divergence-free, which is required to avoid spurious noise sources due to the turbulent inflow in the computational domain. A superposition of anisotropic Gaussian velocity spectra is proposed in Section IV.B to reproduce the key statistics of Kerschen and Gliebe's axisymmetric turbulence model. ${ }^{15}$

\section{IV.A. Isotropic and Anisotropic Gaussian Turbulence}

Gaussian velocity spectra are often used in synthetic turbulence methods ${ }^{12,21,22,31}$ as a simplified but representative approach to model the spectral content of realistic turbulent flows. Section IV.A.1 presents an overview of the advanced digital filter method ${ }^{12}$ for the generation of two-dimensional isotropic turbulence with Gaussian velocity spectra. An extension of this method is presented and validated in Section IV.A.2 to account for variations in the streamwise and lateral length scales of the turbulent flow. 


\section{IV.A.1. Isotropic Gaussian Turbulence}

According to Gea-Aguilera et al.'s work, ${ }^{12}$ which is based on a previous investigation by Dieste and Gabard, ${ }^{49}$ the fluctuating velocity field defined by a two-dimensional isotropic Gaussian eddy is

$$
\begin{aligned}
& u_{x}(\mathbf{x})=-\frac{\epsilon \Delta}{\Lambda^{2}} \sqrt{2 \pi u_{r m s}^{2}}\left(y-y_{e}\right) \exp \left(-\frac{\pi r^{2}}{2 \Lambda^{2}}\right), \\
& u_{y}(\mathbf{x})=+\frac{\epsilon \Delta}{\Lambda^{2}} \sqrt{2 \pi u_{r m s}^{2}}\left(x-x_{e}\right) \exp \left(-\frac{\pi r^{2}}{2 \Lambda^{2}}\right),
\end{aligned}
$$

where $\mathbf{x}=(x, y)$ is a point in the flow field, $\left(x_{e}, y_{e}\right)$ corresponds to the eddy center, $r$ is the distance between a point in the flow field and the eddy center, $u_{r m s}$ is root-mean-square velocity fluctuation, $\Lambda$ is the integral length scale, $\epsilon$ is a random variable that controls the direction of rotation $(+1$ or -1$)$, and $\Delta$ is the separation between eddy centers. Several eddies are introduced through an inlet section into the CAA domain to produce isotropic turbulence. In order to obtain the correct statistics of the turbulent flow, Gea-Aguilera et $a l^{12}$ showed that the eddy radius and the distance between eddy centers should satisfy $r_{e} \geq 3 \Lambda / 2$ and $\Delta \leq \Lambda / 2$, respectively. The method is able to reproduce the isotropic Gaussian energy spectrum proposed by Kraichnan ${ }^{21}$ for two-dimensional turbulence. Therefore, the resulting velocity spectra are defined for the streamwise and transverse fluctuating velocity components as

$$
\begin{aligned}
& \Phi_{11}^{2 \mathrm{D}}\left(k_{x}, k_{y}\right)=\frac{2}{\pi^{3}} u_{r m s}^{2} \Lambda^{4} k_{y}^{2} \exp \left(-\frac{\Lambda^{2} k^{2}}{\pi}\right), \\
& \Phi_{22}^{2 \mathrm{D}}\left(k_{x}, k_{y}\right)=\frac{2}{\pi^{3}} u_{r m s}^{2} \Lambda^{4} k_{x}^{2} \exp \left(-\frac{\Lambda^{2} k^{2}}{\pi}\right),
\end{aligned}
$$

respectively, where $k=\sqrt{k_{x}^{2}+k_{y}^{2}}$ is the overall wavenumber.

\section{IV.A.2. Anisotropic Gaussian Turbulence}

An isotropic Gaussian eddy acts as a spatial filter with the same length scale, $\Lambda$, in each spatial direction. In this section, Eqs. 1 and 2 are extended to account for variations in the streamwise and transverse length scales, $l_{x}$ and $l_{y}$, respectively. Thus, the fluctuating velocity field introduced by an anisotropic Gaussian eddy is defined as

$$
\begin{aligned}
& u_{x}(\mathbf{x})=-\frac{\epsilon \sqrt{\Delta_{x} \Delta_{y}}}{l_{y}^{2}} \sqrt{2 \pi u_{0}^{2}}\left(y-y_{e}\right) \exp \left[-\frac{\pi\left(x-x_{e}\right)^{2}}{2 l_{x}^{2}}\right] \exp \left[-\frac{\pi\left(y-y_{e}\right)^{2}}{2 l_{y}^{2}}\right], \\
& u_{y}(\mathbf{x})=+\frac{\epsilon \sqrt{\Delta_{x} \Delta_{y}}}{l_{x}^{2}} \sqrt{2 \pi u_{0}^{2}}\left(x-x_{e}\right) \exp \left[-\frac{\pi\left(x-x_{e}\right)^{2}}{2 l_{x}^{2}}\right] \exp \left[-\frac{\pi\left(y-y_{e}\right)^{2}}{2 l_{y}^{2}}\right],
\end{aligned}
$$

where $u_{0}$ is a characteristic velocity term that controls the amplitude of the eddies. The same value of $u_{0}$ is used for both velocity components as a consequence of the divergence-free condition that is imposed on each eddy. It should be noted that the equations for an isotropic Gaussian eddy are recovered by setting $u_{0}=u_{r m s}, l_{x}=l_{y}=\Lambda$, and $\Delta_{x}=\Delta_{y}=\Delta$ in Eqs. 5 and 6 . Figure 2 shows contours and streamlines of the fluctuating velocity field introduced by an anisotropic Gaussian eddy. This type of eddy introduces streamlines with the shape of an ellipse, i.e., the eddies are stretched in a prevailing direction. In contrast, isotropic Gaussian eddies introduce circular-shaped streamlines with a constant radius.

Similarly to the implementation strategy of the advanced digital filter method for isotropic turbulence, ${ }^{12}$ eddies are introduced into the CAA domain through a local inlet section to generate anisotropic turbulence. Figure 3 shows a schematic representation of the numerical implementation. The correct statistics of the anisotropic turbulent flow are recovered provided that the separation between eddy centers are $\Delta_{x} \leq l_{x} / 2$ and $\Delta_{y} \leq l_{y} / 2$, and the eddy radii are $r_{e, x} \geq 3 l_{x} / 2$ and $r_{e, y} \geq 3 l_{y} / 2$. These limiting values are based on those presented for isotropic turbulence in Section IV.A.1. Since the eddies are convected as frozen turbulence ${ }^{50}$ by the mean flow, no additional control on the eddy properties is possible once injected into the CAA domain. The assumption of frozen turbulence is often used for leading edge noise predictions, especially in 
the context of aero-engine broadband noise. ${ }^{11,49} \mathrm{~A}$ new set of anisotropic Gaussian eddies are injected into the CAA domain every $\Delta_{x} /\left(U_{x} \mathrm{~d} t\right)$ iterations, where $U_{x}$ is the freestream speed and $\mathrm{d} t$ is the time step of the simulation. This ensures that the separation between eddy centers in the streamwise direction is $\Delta_{x}$. The length of the inlet section in the $y$-direction is at least $2 r_{e, y}$, which guarantees the correct turbulence statistics along the center line downstream of the inlet section. For instance, for the symmetric airfoil configuration that is shown in Figure 3, the inlet section length should be of at least $2 r_{e, y}+t_{A}$, where $t_{A}$ is the maximum thickness of the airfoil.

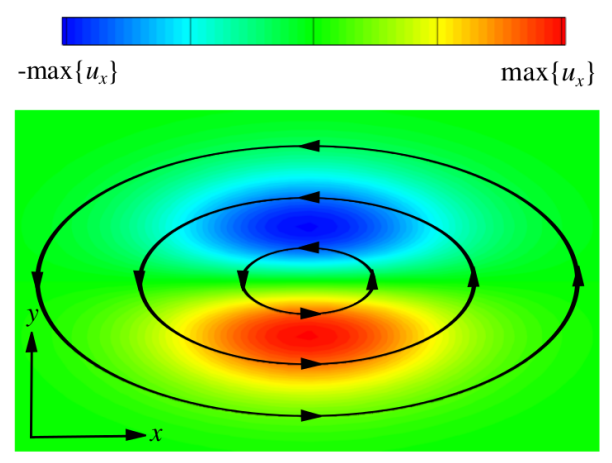

(a) $u_{x}$ contours and streamlines.

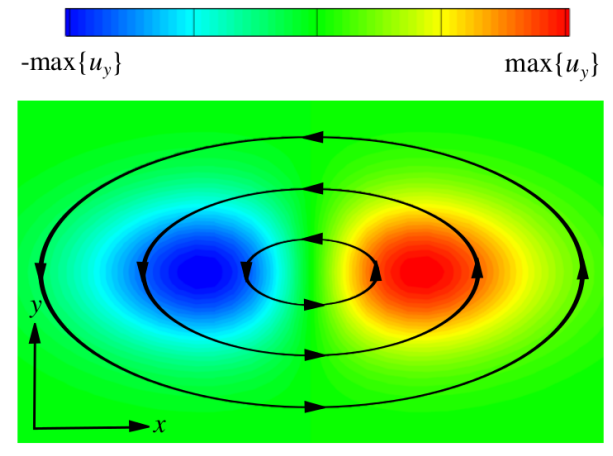

(b) $u_{y}$ contours and streamlines.

Figure 2. Fluctuating velocity field due to a two-dimensional anisotropic Gaussian eddy with $l_{x}=3 l_{y}$.
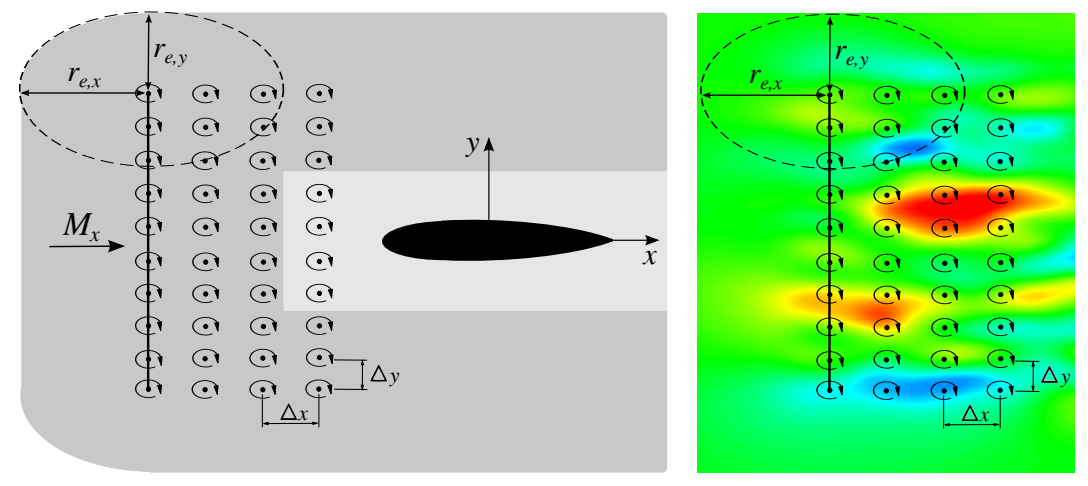

Figure 3. Inlet section and parameters to introduce anisotropic turbulence. The size of the eddies is controlled by $r_{e, x}$ and $r_{e, y}$, and the distance between eddy centers relies on $\Delta x$ and $\Delta y$.

The spectral content of turbulent flows generated by using anisotropic Gaussian eddies is defined by the streamwise and transverse velocity spectra

$$
\begin{aligned}
& \Phi_{11}^{2 \mathrm{D}}\left(k_{x}, k_{y}\right)=\frac{2}{\pi^{3}} u_{0}^{2} l_{x}^{2} l_{y}^{2} k_{y}^{2} \exp \left(-\frac{l_{x}^{2} k_{x}^{2}}{\pi}\right) \exp \left(-\frac{l_{y}^{2} k_{y}^{2}}{\pi}\right), \\
& \Phi_{22}^{2 \mathrm{D}}\left(k_{x}, k_{y}\right)=\frac{2}{\pi^{3}} u_{0}^{2} l_{x}^{2} l_{y}^{2} k_{x}^{2} \exp \left(-\frac{l_{x}^{2} k_{x}^{2}}{\pi}\right) \exp \left(-\frac{l_{y}^{2} k_{y}^{2}}{\pi}\right),
\end{aligned}
$$

respectively, where $\left(k_{x}, k_{y}\right)$ correspond to the wavenumber components. The integration of each velocity spectrum over the whole range of both $k_{x}$ and $k_{y}$ leads to the mean-square velocities, which are given by $u_{x, r m s}^{2}=u_{0}^{2} l_{x} / l_{y}$ and $u_{y, r m s}^{2}=u_{0}^{2} l_{y} / l_{x}$. Hence, the ratio of root-mean-square velocity components is proportional to the ratio of length scales, $u_{x, r m s} / u_{y, r m s}=l_{x} / l_{y}$. This relationship, which is a consequence of the divergence-free condition, shows that it is only possible to set random values for three of the four parameters $u_{x, r m s}, u_{y, r m s}, l_{x}$ and $l_{y}$.

For validation purposes, two simulations were run using the digital filter method to generate anisotropic Gaussian turbulence. The numerical implementation was validated using $u_{0}^{2}=3 \mathrm{~m}^{2} / \mathrm{s}^{2}$, with stretched turbulence in the streamwise $\left(l_{x}=3 l_{y}\right.$ with $\left.l_{y}=0.004 \mathrm{~m}\right)$ and transverse $\left(l_{y}=3 l_{x}\right.$ with $\left.l_{x}=0.004 \mathrm{~m}\right)$ directions. Anisotropic Gaussian eddies were injected into a CAA mesh with a constant grid spacing of 
$0.001 \mathrm{~m}$ in a $M_{x}=0.3$ uniform mean flow. A monitor point was placed within the turbulent flow, $0.018 \mathrm{~m}$ downstream of the inlet section, to collect $3.4 \times 10^{4}$ velocity samples every 3 non-dimensional time steps $\mathrm{d} t c_{0} / L_{r e f}=4 \times 10^{-4}$. One-dimensional spectra for the streamwise and transverse velocity components are defined as

$$
\begin{aligned}
& E_{11}^{2 \mathrm{D}}\left(k_{x}\right)=2 \int_{-\infty}^{+\infty} \Phi_{11}^{2 \mathrm{D}}\left(k_{x}, k_{y}\right) \mathrm{d} k_{y}, \\
& E_{22}^{2 \mathrm{D}}\left(k_{x}\right)=2 \int_{-\infty}^{+\infty} \Phi_{22}^{2 \mathrm{D}}\left(k_{x}, k_{y}\right) \mathrm{d} k_{y},
\end{aligned}
$$

respectively. Figure 4 shows analytical and numerical one-dimensional spectra ${ }^{\text {a }}$ obtained from velocity samples using Thomson's multitaper spectral analysis. ${ }^{51}$ An agreement of better than $1.5 \mathrm{~dB}$ is found at all frequencies.

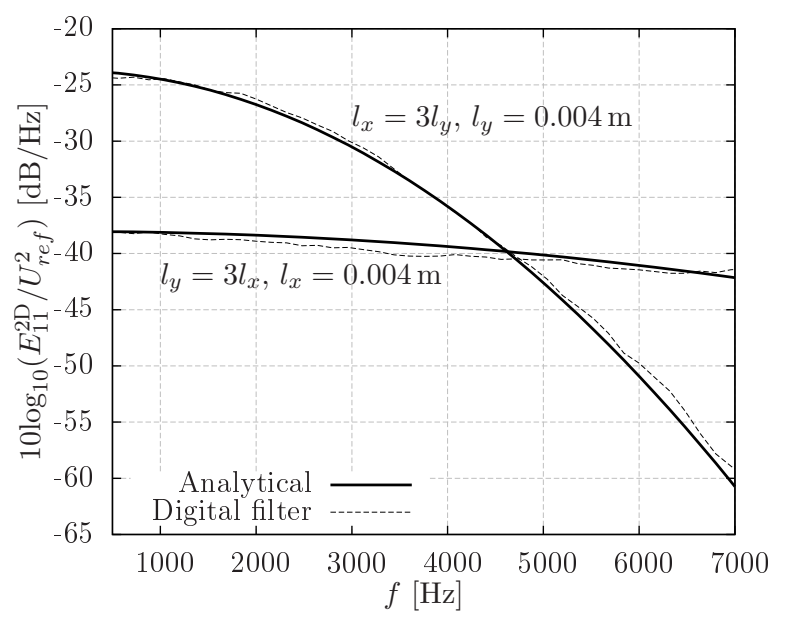

(a) Streamwise spectrum.

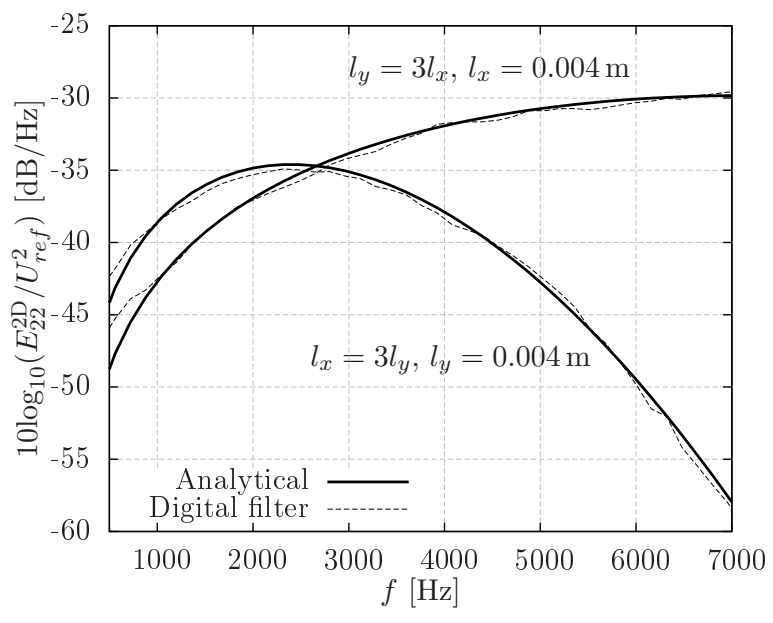

(b) Transverse spectrum.

Figure 4. One-dimensional spectra of anisotropic Gaussian turbulence.

\section{IV.B. Anisotropic Non-Gaussian Turbulence}

\section{IV.B.1. Anisotropic Gaussian Superposition}

The superposition of Gaussian spectra is useful to expand the spectral content of a turbulent flow. This technique has been used successfully in Fourier mode methods, ${ }^{23,24}$ and digital filter methods. ${ }^{12,32}$ Using an advanced digital filter method for isotropic turbulence, Gea-Aguilera et al. ${ }^{12}$ proposed to define a new eddy shape from a superposition of Gaussian eddies. This was possible due to the relationship between the equation of the spatial filter in wavenumber space and the isotropic energy spectrum, which is related to the average kinetic energy at a particular length scale in any spatial direction. This strategy cannot be followed for anisotropic turbulence, in which the energy spectrum is not defined. In this work, generalized anisotropic velocity spectra are obtained through a linear summation of $N_{e}$ anisotropic Gaussian velocity spectra, i.e.,

$$
\Phi_{i j}^{s u m}=\sum_{k=1}^{N_{e}} \Phi_{i j, k},
$$

where $\Phi_{i j, k}$ is defined for the streamwise and transverse velocity spectra by Eqs. 7 and 8 , respectively. Anisotropic Gaussian velocity spectra require the definition of an amplitude term, $u_{0, i}$, and two length scales, $l_{x, i}$ and $l_{y, i}$. These are case-dependent parameters that rely on both the non-Gaussian velocity spectrum and the frequency range to be matched.

A setup based on the numerical methodology shown in Figure 3 is followed in order to simplify the inlet section implementation. A total number, $N_{e}$, of anisotropic Gaussian eddies with different $u_{0, i}, l_{x, i}, l_{y, i}$ are

\footnotetext{
${ }^{\text {a }}$ For frozen turbulence, $E_{11}(f)=2 \pi E_{11}\left(k_{x}\right) / U_{x}$ and $E_{22}(f)=2 \pi E_{22}\left(k_{x}\right) / U_{x}$.
} 
introduced simultaneously at each eddy center along the inlet section. Limiting values for the eddy radius and separation between eddy centers are based on the largest and shortest length scales involved in the anisotropic Gaussian superposition. Thus, the correct statistics of the turbulent flow are obtained provided that $r_{e, x} \geq 3 \max \left\{l_{x, i}\right\} / 2, r_{e, y} \geq 3 \max \left\{l_{y, i}\right\} / 2, \Delta_{x} \leq \min \left\{l_{x, i}\right\} / 2$ and $\Delta_{y} \leq \min \left\{l_{y, i}\right\} / 2$. The fluctuating velocity field introduced by the summation of $N_{e}$ eddies at each eddy center is defined as

$$
\begin{aligned}
& u_{x}(\mathbf{x})=-\sqrt{2 \pi \Delta_{x} \Delta_{y}}\left(y-y_{e}\right) \sum_{i=1}^{N_{e}} \frac{\epsilon_{i} u_{0, i}}{l_{y, i}^{2}} \exp \left[-\frac{\pi\left(x-x_{e}\right)^{2}}{2 l_{x, i}^{2}}\right] \exp \left[-\frac{\pi\left(y-y_{e}\right)^{2}}{2 l_{y, i}^{2}}\right], \\
& u_{y}(\mathbf{x})=+\sqrt{2 \pi \Delta_{x} \Delta_{y}}\left(x-x_{e}\right) \sum_{i=1}^{N_{e}} \frac{\epsilon_{i} u_{0, i}}{l_{x, i}^{2}} \exp \left[-\frac{\pi\left(x-x_{e}\right)^{2}}{2 l_{x, i}^{2}}\right] \exp \left[-\frac{\pi\left(y-y_{e}\right)^{2}}{2 l_{y, i}^{2}}\right] .
\end{aligned}
$$

\section{IV.B.2. Homogeneous Axisymmetric Turbulence}

A simple description of anisotropic turbulence corresponds to homogeneous axisymmetric turbulence, ${ }^{38}$ in which the statistics of the turbulent flow are invariant to rotations along a preferred axis. Additionally, axisymmetric turbulence has been reported experimentally in both fan intakes ${ }^{15}$ and fan wakes, ${ }^{17}$ and used in previous works in the context of leading edge noise ${ }^{7}$ and turbofan engine broadband noise. ${ }^{20}$ In this work, leading edge noise predictions of isolated airfoils are performed by using the homogeneous axisymmetric turbulence model of Kerschen and Gliebe. ${ }^{15}$ Assuming that the axisymmetric axis is aligned with the $x$ direction, i.e. the mean flow, the three-dimensional axial (streamwise) and transverse velocity spectra are defined as

$$
\Phi_{11}\left(k_{x}, k_{y}, k_{z}\right)=\frac{2 l_{a} l_{t}^{4} u_{a}^{2}\left(k_{y}^{2}+k_{z}^{2}\right)}{\pi^{2}\left[1+l_{a}^{2} k_{x}^{2}+l_{t}^{2}\left(k_{y}^{2}+k_{z}^{2}\right)\right]^{3}},
$$

and

$$
\Phi_{22}\left(k_{x}, k_{y}, k_{z}\right)=\frac{2 l_{a} l_{t}^{4} u_{a}^{2}}{\pi^{2}\left[1+l_{a}^{2} k_{x}^{2}+l_{t}^{2}\left(k_{y}^{2}+k_{z}^{2}\right)\right]^{3}}\left[k_{x}^{2}+k_{z}^{2}\left(2 \frac{u_{t}^{2}}{u_{a}^{2}}-\frac{l_{t}^{2}}{l_{a}^{2}}\right)\right],
$$

respectively, where $l_{a}, u_{a}$ and $l_{t}, u_{t}$ are the length scale and root-mean-square of the fluctuating velocity component in the axial and transverse directions, respectively, and $\left(k_{x}, k_{y}, k_{z}\right)$ correspond to the wavenumber components. A constraint of this model is that $2\left(u_{t} / u_{a}\right)^{2} \geq\left(l_{t} / l_{a}\right)^{2}$, which is easily verified in fan wake experiments. Furthermore, velocity spectra corresponding to the isotropic Liepmann turbulence model ${ }^{52}$ are recovered by setting $l_{a}=l_{t}=\Lambda$ and $u_{a}=u_{t}=u_{r m s}$ in Eqs. 14 and 15. This reinforces the use of Kerschen and Gliebe's axisymmetric turbulence model ${ }^{15}$ for broadband noise predictions of turbulence-airfoil interaction, since it can better reflect the energy contained in a wider range of length scales than a single Gaussian spectrum.

\section{Effects of Anisotropic Turbulence on Leading Edge Noise}

In this section, leading edge noise is investigated using isolated airfoils in moderately anisotropic turbulent flows. Variations in freestream Mach number, airfoil thickness and angle of attack are explored. For each airfoil configuration, the study examines effects on noise due to changes in streamwise-to-transverse length scale ratios, $l_{a} / l_{t}$, between 0.33 and 3 using Kerschen and Gliebe's axisymmetric turbulence model. ${ }^{15}$ In ducted-fan configurations and open-jet wind tunnel experiments, turbulent flows tend to be stretched in the axial direction, so that cases with $l_{a} / l_{t}>1$ are particularly relevant for turbofan engine applications. Nevertheless, cases with $l_{a} / l_{t}<1$ are also documented for completeness of the analysis. In this work, turbulence length scales are small compared to the airfoil chord, as is the case in typical fan wakes.

The CAA methodology used herein for leading edge noise predictions is similar to the 'pseudo threedimensional turbulence' approach proposed by Gea-Aguilera et al. ${ }^{12}$ The objective of such an approach is to combine affordable two-dimensional CAA simulations with a three-dimensional Ffowcs-Williams and Hawkings (FW-H) solver to perform leading edge noise predictions that can be compared with experimental results from open-jet wind tunnels.

For the two-dimensional CAA simulations, the anisotropic Gaussian superposition methodology presented in Section IV.B.1 is applied to synthesize homogeneous axisymmetric turbulence under the assumption of $k_{z}=0$. This assumption is based on Amiet's flat plate model, ${ }^{3}$ in which the spanwise wavenumber $\left(k_{z}\right.$ 
with the notation used in this work) of the oncoming turbulence does not contribute to the broadband noise for an observer at the mid-span plane in the far-field, provided that the turbulence length scale is small in comparison to the airfoil span. Thus, the $k_{z}=0$ assumption is used to generate a two-dimensional turbulent flow with the key statistics of three-dimensional homogeneous axisymmetric velocity spectra presented in Section IV.B.2.

The baseline turbulent flow corresponds to isotropic Liepmann turbulence with $l_{a}=l_{t}$ set to the integral length scale $\Lambda=0.008 \mathrm{~m}$ and $u_{a}=u_{r m s}$ set to $1.7 \%$ of the freestream speed. The $k_{z}=0$ hypothesis removes $u_{t}$ from Eq. 15. Nevertheless, $u_{t}$ is assumed to take a value that satisfies the constraint $2\left(u_{t} / u_{a}\right)^{2} \geq\left(l_{t} / l_{a}\right)^{2}$ for the validity of the model. Moderate variations in the length scales away from the baseline case are made to study the effects of anisotropic turbulence on symmetric thick airfoils. Figure 5 shows a schematic representation of the airfoil configurations and reference frame that is used for the noise predictions. Each CAA mesh extends to 4 chordlengths in all directions around the airfoils, which have a chord of $c=0.15 \mathrm{~m}$. The grid resolution is at least 8 points-per-wavelength for the smallest vortical waves that are correctly propagated by the CAA code. This corresponds to a chord-based Strouhal number, $f c / U_{x}$, of up to 6 for the grid of a NACA 0001 airfoil at zero angle of attack in a $M_{x}=0.6$ flow. Such a configuration is used for validation of the numerical methodology. A refined grid with a frequency resolution up to $f c / U_{x}=12$ is used to study more challenging airfoil configurations on a NACA 0012 airfoil.

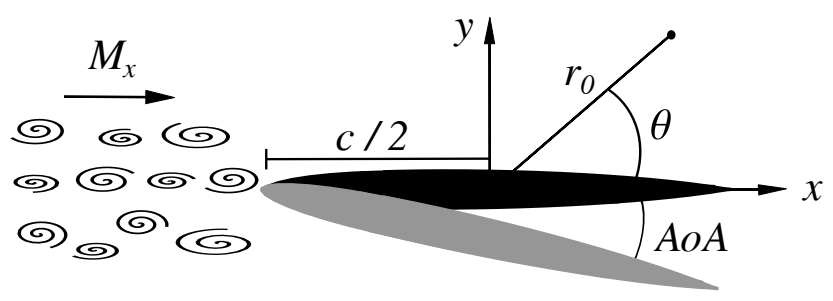

Figure 5. Reference frame, far-field observers and rotation around the leading edge for the angle of attack study.

To allow far-field noise predictions, unsteady data are collected on the airfoil surface from the twodimensional CAA simulation and copied repeatedly along the airfoil span. In the current study, the airfoil semi-span is set to $d=0.225 \mathrm{~m}$. This numerical methodology generates a three-dimensional airfoil with straight leading edge that is used in a three-dimensional FW-H solver based on Farassat and Succi's formulation 1A. ${ }^{53}$ The FW-H solver radiates the sound in a uniform mean flow to the far-field at a distance of $r_{0}=20 c$ in the mid-span plane. Time-dependent pressure fluctuations are calculated at 360 observer locations, with an observation angle resolution of $1^{\circ}$. The unsteady pressure in the far-field is used to compute the sound power spectrum by assuming cylindrical noise radiation, ${ }^{4}$

$$
\mathcal{P}(f)=\frac{d r_{0}}{\rho_{0} c_{0}} \int_{0}^{2 \pi} S_{p p}\left(r_{0}, \theta, f\right) \frac{\beta^{4} A(\theta, M)}{[A(\theta, M)-M \cos \theta]^{2}} \mathrm{~d} \theta,
$$

where $S_{p p}$ is the pressure spectral density, $\beta=\sqrt{1-M_{x}^{2}}$ and $A(\theta, M)=\sqrt{1-M_{x}^{2} \sin ^{2} \theta}$. The pressure spectral density is obtained by using Thomson's multitaper spectral analysis. ${ }^{51}$ Note that due to the $k_{z}=0$ assumption in the velocity spectra, a $\pi / d$ scaling factor needs to be applied to all frequencies of the pressure spectral density in order to recover the correct amplitude of the noise predictions.

Analysis of the numerical simulations is organized as follows. Section V.A presents CAA simulations of a NACA 0001 airfoil at zero angle of attack for validation of turbulence and noise spectra. In Section V.B, airfoil thickness effects on noise are studied for a NACA 0012 airfoil at zero angle of attack, in various freestream Mach number flows, when encountering both isotropic and anisotropic turbulence. This airfoil is placed at an angle of attack of $6^{\circ}$ in Section V.C.

\section{V.A. Flat Plates and Thin Airfoils at Zero Angle of Attack}

Simulations of a NACA 0001 airfoil are presented for validation of the leading edge noise prediction methodology and initial analysis of noise from thin airfoils in anisotropic turbulence. The airfoil is placed in a $M_{x}=0.6$ uniform mean flow that convects the turbulent inflow as frozen turbulence. ${ }^{50}$ The anisotropic Gaussian superposition methodology is used to reproduce Kerschen and Gliebe's axisymmetric turbulence model $^{15}$ with $k_{z}=0$. Appendix A presents values for $u_{0, i}, l_{x, i}$ and $l_{y, i}$ in anisotropic Gaussian spectra that are used in the superposition. 
To assess that the correct velocity spectra are recovered from the CAA simulations, a monitor point was placed at approximately $0.2 \mathrm{c}$ upstream of the airfoil leading edge. At this location, a total of $3 \times 10^{4}$ samples of velocity fluctuations were recorded every 283 non-dimensional time steps $\mathrm{d} t c_{0} / L_{r e f}=4 \times 10^{-6}$. Figure 6 shows numerical and analytical one-dimensional spectra of anisotropic turbulence for moderate $l_{a} / l_{t}$ ratios. Results are in good agreement, showing the effectiveness of the anisotropic Gaussian superposition methodology.

Analytical $E_{11}$ and $E_{22}$ show a similar behavior with variations in the length scales with respect to the isotropic turbulence baseline. An increase in the streamwise length scale, $l_{a} / l_{t}>1$, leads to slightly higher amplitudes at low frequencies and a significant reduction at high frequencies. Additionally, $E_{22}$ suffers from a slight shift of the maximum value towards lower frequencies. In contrast, an increase of the transverse length scale, $l_{a} / l_{t}<1$, leads to a nearly constant increase in amplitude at all frequencies.

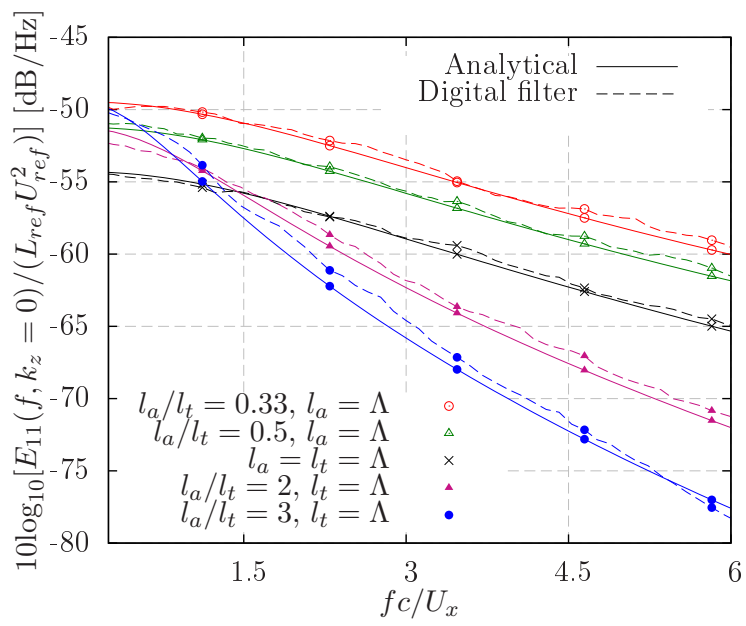

(a) Streamwise.

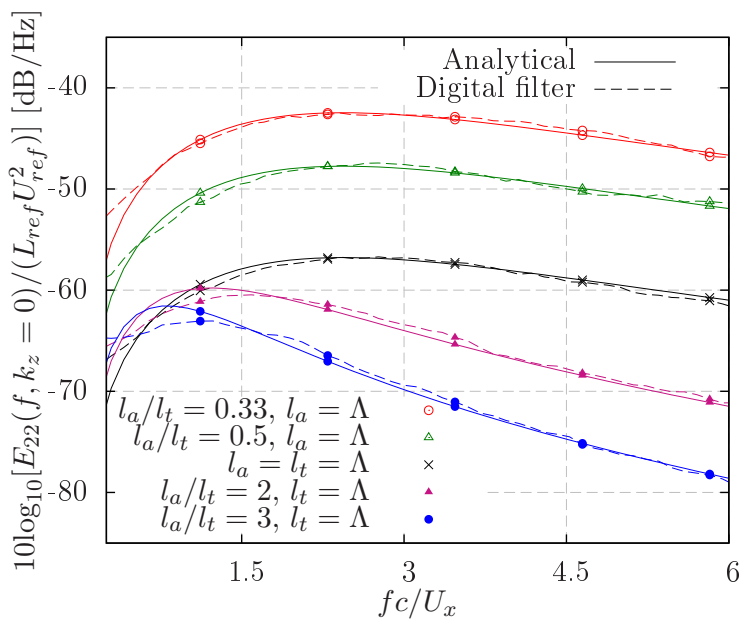

(b) Transverse.

Figure 6. One-dimensional spectra of homogeneous axisymmetric turbulence.

The use of axisymmetric turbulence produces a redistribution of the energy in the velocity spectra when compared to isotropic turbulence with the same kinetic energy. This redistribution of the energy, which is caused by the different length scales involved, changes the amount of energy that contributes to the turbulence-airfoil interaction noise. The use of anisotropic turbulence can potentially lead to more accurate leading edge noise predictions. This is particularly important for analytical models, in which noise predictions are quite sensitive to the input turbulence model, as reported in Posson et al.'s work. ${ }^{20}$

Figure 7 shows analytical and numerical PWL and SPL spectra of a NACA 0001 airfoil. For validation purposes, Amiet's theory ${ }^{3}$ for a flat plate is used with the transverse velocity spectrum in Eq. 15. The agreement between numerical and analytical predictions is within $2 \mathrm{~dB}$ at all frequencies. In Posson et al.'s analytical model for flat plate cascades, ${ }^{20}$ a decrease in noise levels and a shift of the maximum noise amplitude towards lower frequencies was found when using Kerschen and Gliebe's axisymmetric turbulence ${ }^{15}$ with $l_{a} / l_{t}=2$ instead of $l_{a} / l_{t}=1$. This result agrees with the trends of the PWL spectra in Figure 7 for an isolated flat plate with $l_{a} / l_{t}=2$.

The shape of the PWL spectra in Figure 7 closely follow those of the $E_{22}$ spectra in Figure 6, reaching the peak value at the same chord-based Strouhal number for each anisotropic turbulence case. Furthermore, the amplitude separation at a given frequency in $\mathrm{dB}$ between two particular $l_{a} / l_{t}$ ratios is similar in PWL and $E_{22}$ spectra. Amiet ${ }^{3}$ showed that the broadband noise due to the interaction of isotropic turbulence with a flat plate only depends on the transverse velocity spectrum. This result, which has previously been assessed in numerical simulation of symmetric NACA airfoils with $1 \%$ and $2 \%$ thickness-to-chord ratio, ${ }^{11,12}$ remains valid for thin airfoils in anisotropic turbulence.

Directivity patterns at $f c / U_{x}=3$ are shown in Figure 8 for further validation of the CAA simulations. The agreement of the numerical results with Amiet's model ${ }^{3}$ prediction is better than $2 \mathrm{~dB}$ at the majority of observer angles. The location of the lobes due to the loss of compactness at high frequencies is correctly predicted by the numerical simulations. 


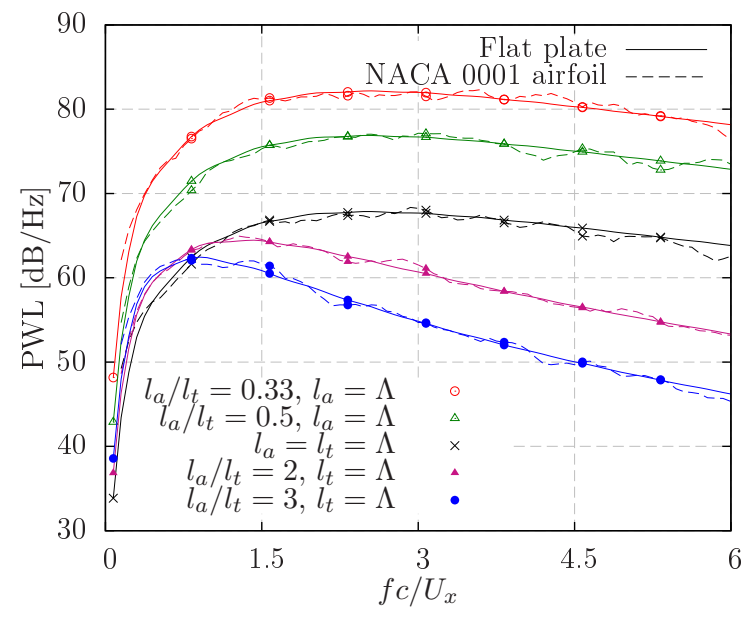

(a) PWL spectra.

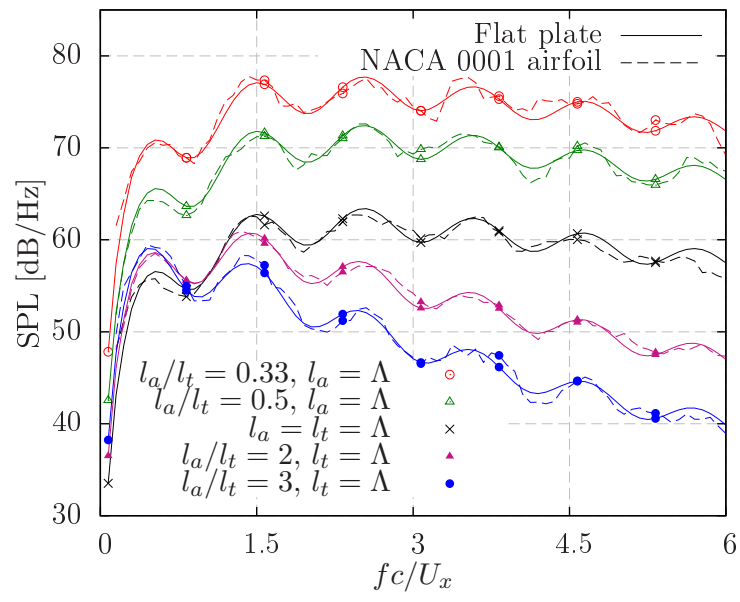

(b) SPL spectra at $\theta=90^{\circ}$.

Figure 7. PWL and SPL spectra for a NACA 0001 airfoil in anisotropic turbulence.

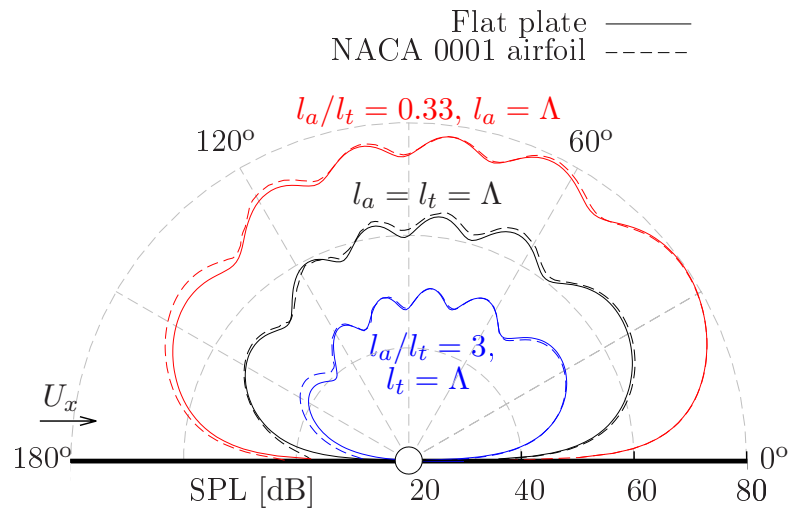

(a) $f c / U_{x}=3$.

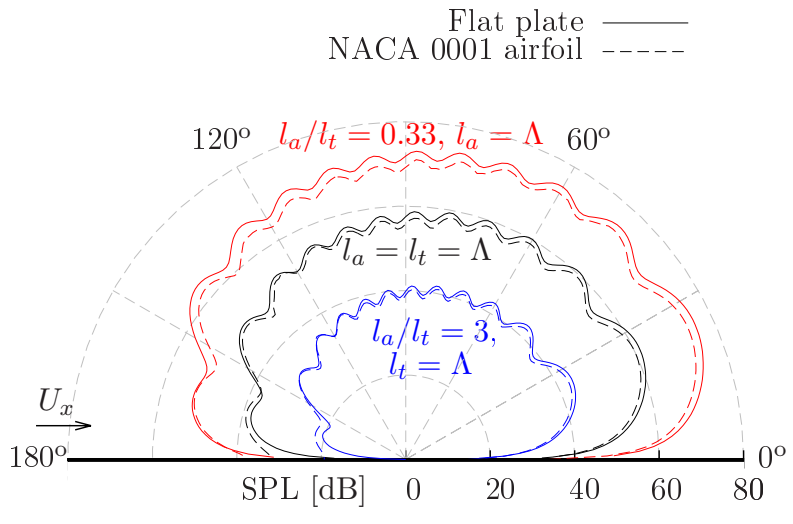

(b) $f c / U_{x}=6$.

Figure 8. Directivity plots for a NACA 0001 airfoil in anisotropic turbulence.

\section{V.B. Thick Airfoils at Zero Angle of Attack in Various Freestream Mach Number Flows}

The effects of airfoil thickness on noise are studied for a NACA 0012 airfoil at zero angle of attack. It is well known that uniform mean flows over-predict leading edge noise from thick airfoils at high frequencies, and that viscosity can be neglected without significant loss of accuracy in the noise predictions. ${ }^{8,54}$ Consequently, an inviscid mean flow is used in the CAA simulations to account for mean flow gradients around the NACA 0012 airfoil. This allows the turbulent structures to be distorted in the vicinity of the airfoil leading edge, which is essential for correct leading edge noise predictions of thick airfoils. ${ }^{54}$ CAA simulations were run at two different freestream Mach numbers, $M_{x}=0.3$ and $M_{x}=0.6$, using the parameters in Appendix A for the anisotropic Gaussian superposition.

Figure 9 shows instantaneous contours of non-dimensional vorticity magnitude and fluctuating pressure in CAA simulations with eddies stretched in the streamwise and transverse directions. In both cases, acoustic pressure is generated at the airfoil leading edge and radiated to the far-field with a dipole-like directivity. However, pressure contours due to anisotropic turbulence with $l_{a} / l_{t}=0.33$ present a broader frequency content than with $l_{a} / l_{t}=3$. This observation is related to the shape of one-dimensional spectra shown in Figure 6, in which the small amplitude difference between high and low frequencies in the $l_{a} / l_{t}=0.33$ case makes fluctuating pressure contours to show a wide frequency content. In contrast, most energy in one-dimensional spectra with $l_{a} / l_{t}=3$ concentrates at low frequencies.

Figure 10 shows PWL spectra from anisotropic turbulence interacting with a NACA 0012 airfoil in a 


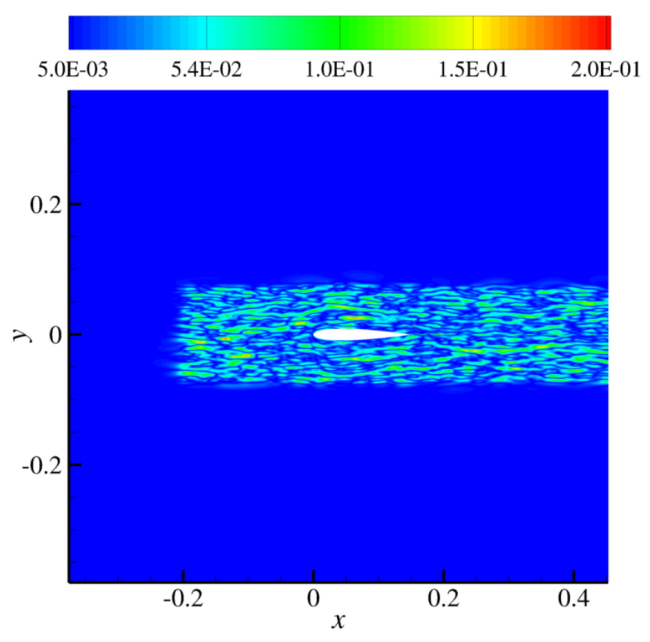

(a) Non-dimensional vorticity magnitude with $l_{a} / l_{t}=3$.

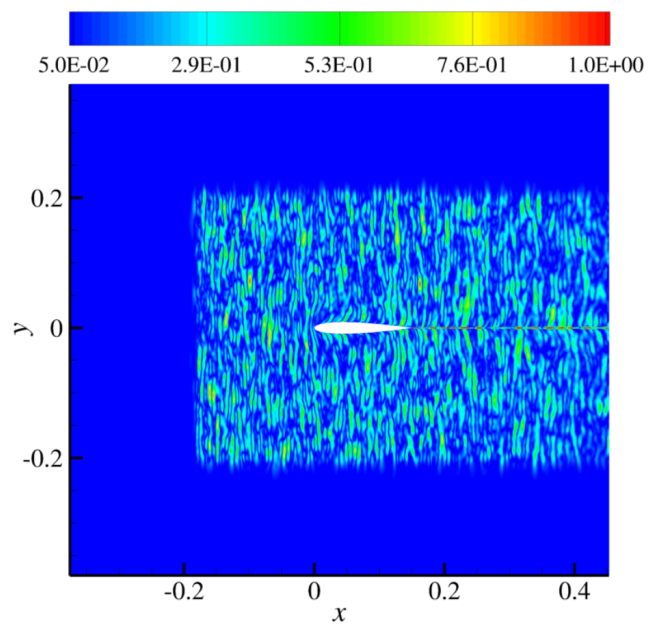

(c) Non-dimensional vorticity magnitude with $l_{a} / l_{t}=$ 0.33 .

Figure 9. Contour plots of a NACA 0012 airfoil interacting with anisotropic synthetic turbulence at $M_{x}=0.3$.

$M_{x}=0.3$ flow compared with analytical predictions for a flat plate. Noise reduction due to airfoil thickness occurs in all anisotropic turbulence cases investigated, following the same trends as the isotropic turbulence baseline. Therefore, the amount of noise reduction due to thickness is independent of the anisotropy in this case. Comparison of numerical PWL spectra with the flat plate prediction ${ }^{3}$ shows a good overlap in $\Delta \mathrm{PWL}=\left.\mathrm{PWL}\right|_{\text {flat plate }}-\left.\mathrm{PWL}\right|_{\mathrm{NACA} 0012}$, which quantifies the noise reduction. Paterson and Amiet ${ }^{5}$ found experimentally a $5 \mathrm{~dB}$ noise reduction on a NACA 0012 airfoil at $f t_{A} / U_{x}=1$, which was used as a reference value for the breakdown of the flat plate model. This limiting frequency corresponds to $f c / U_{x}=8.3$ in Figure 10. An earlier appearance of airfoil thickness effects on noise from a NACA 0012 airfoil has been reported in recent numerical and experimental studies. ${ }^{54,55}$ In this work, PWL spectra for isotropic turbulence suggests that the breakdown occurs at a lower frequency than predicted by Paterson and Amiet. ${ }^{5} \mathrm{~A} 5 \mathrm{~dB}$ reduction in PWL due to thickness effect of a NACA 0012 airfoil is found at approximately $f c / U_{x}=7$ for both isotropic and moderate anisotropic turbulence at $M_{x}=0.3$.

PWL spectra from simulations with a freestream Mach number of 0.6 are presented in Figure 11. $\Delta$ PWL spectra scales linearly with $f c / U_{x}$, as found for $M_{x}=0.3$ in Figure 10. This result is consistent with Paruchuri et al.'s work ${ }^{55}$ for thick airfoils in isotropic turbulence, in which $\Delta \mathrm{PWL}$ was found to scale linearly with $f / U_{x}$.

The increase in the mean flow Mach number results in a worse overlap of $\Delta \mathrm{PWL}$ spectra for different 


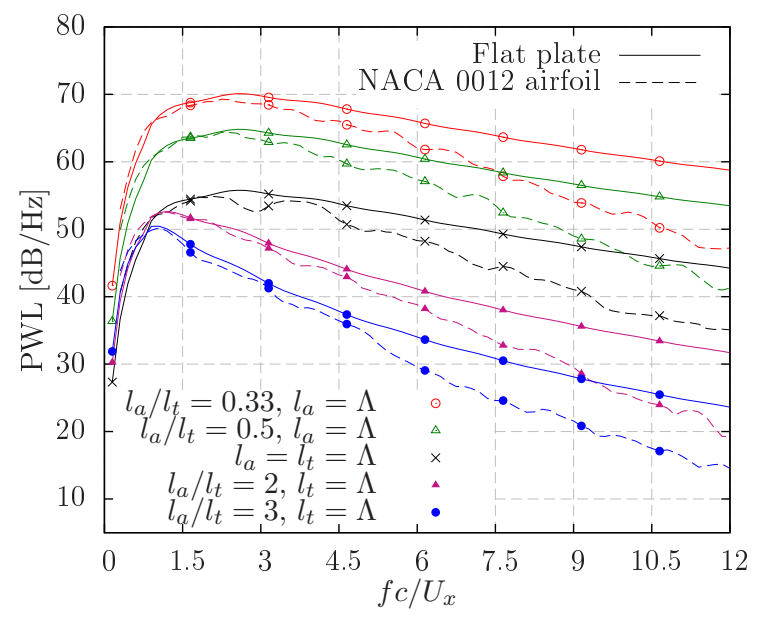

(a) PWL spectra.

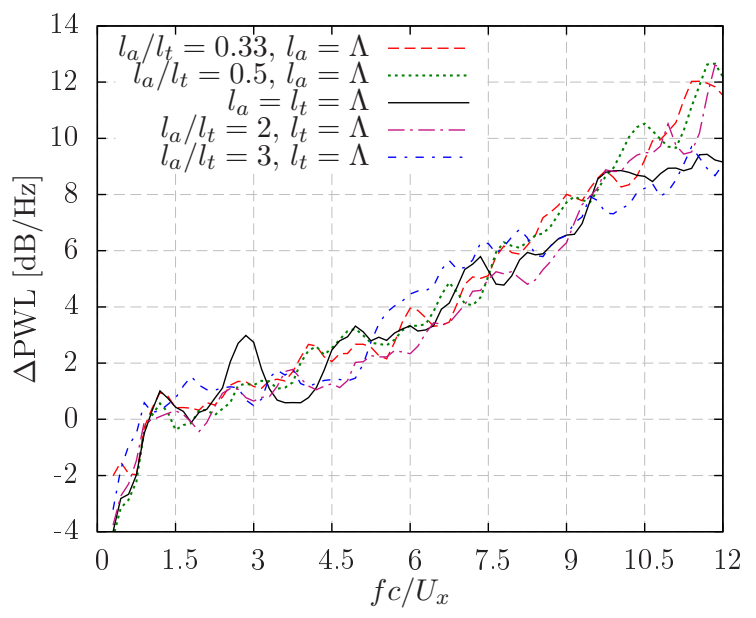

(b) $\Delta \mathrm{PWL}=\left.\mathrm{PWL}\right|_{\text {flat plate }}-\left.\mathrm{PWL}\right|_{\text {NACA } 0012}$.

Figure 10. Noise spectra from anisotropic turbulence interacting with a NACA 0012 airfoil at $M_{x}=0.3$ and $A \circ A=0^{\circ}$.

anisotropy levels in Figure 11. Particularly, $\left.\Delta \mathrm{PWL}\right|_{l_{a} / l_{t}>1}$ is consistently lower than $\left.\Delta \mathrm{PWL}\right|_{l_{a} / l_{t}<1}$. In other words, cases with $l_{a} / l_{t}>1$ tend to be about $2-3 \mathrm{~dB}$ louder at all frequencies than cases with $l_{a} / l_{t}<1$ when compared to their corresponding flat plate predictions. Gill et al. ${ }^{11}$ showed that the transverse fluctuating velocity component is mainly responsible for the leading edge noise of a thick airfoil at zero angle of attack in isotropic turbulence. However, the streamwise fluctuating velocity component becomes increasingly important in anisotropic turbulence with $l_{a} / l_{t}>1$, which may delay the onset of airfoil thickness effects on leading edge noise at high Mach numbers.

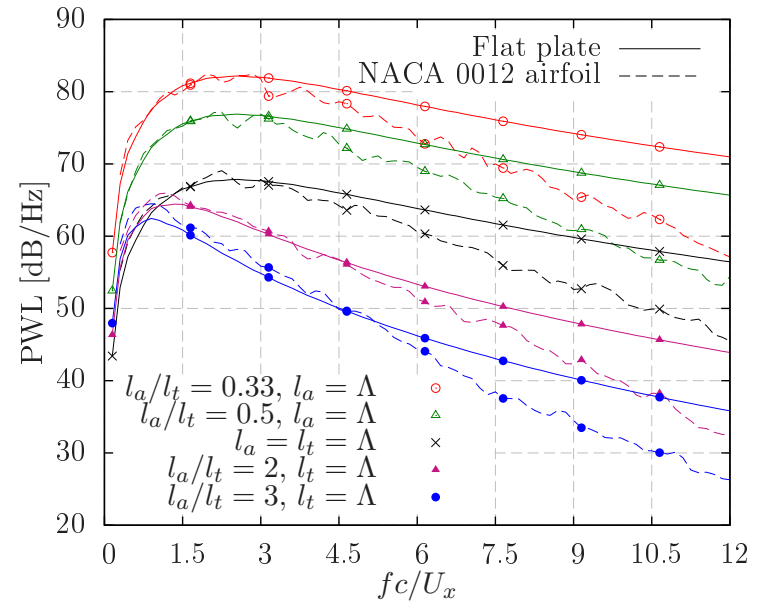

(a) PWL spectra.

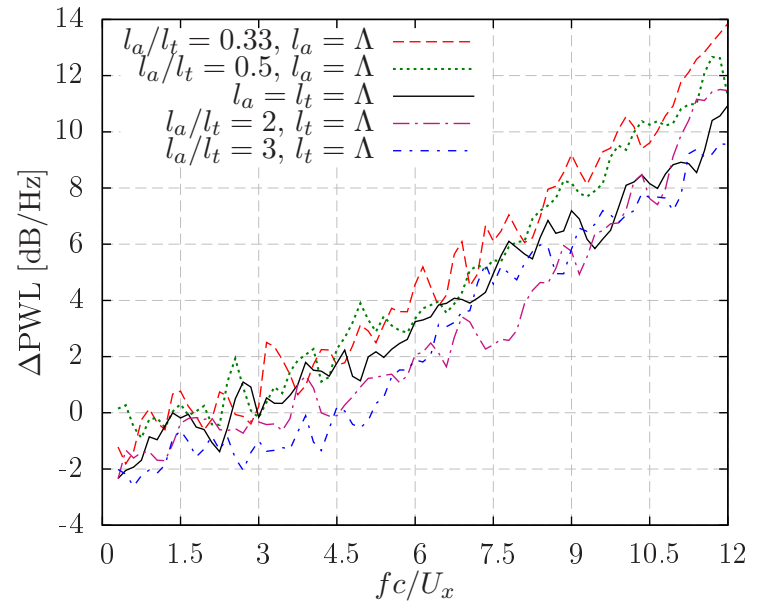

(b) $\Delta \mathrm{PWL}=\left.\mathrm{PWL}\right|_{\text {flat plate }}-\left.\mathrm{PWL}\right|_{\text {NACA } 0012}$.

Figure 11. Noise spectra from anisotropic turbulence interacting with a NACA 0012 airfoil at $M_{x}=0.6$ and $A o A=0^{\circ}$.

\section{V.C. Thick Airfoils at Non-zero Angle of Attack}

In this section, the NACA 0012 airfoil is rotated $6^{\circ}$ around its leading edge, as shown in Figure 5. An inviscid mean flow with freestream Mach number $M_{x}=0.3$ is used in CAA simulations. This airfoil configuration is suitable to study possible effects of angle of attack on noise, while ensuring a subsonic flow around the airfoil. Figure 12 shows PWL spectra of a NACA 0012 airfoil at non-zero angle of attack.

Noise predictions are compared against simulations at $A o A=0^{\circ}$ from Section V.B by using a redefined $\triangle \mathrm{PWL}=\left.\mathrm{PWL}\right|_{A o A=6^{\circ}}-\left.\mathrm{PWL}\right|_{A o A=0^{\circ}} . \Delta \mathrm{PWL}$ spectra shows that an increase in angle of attack tends to reduce the noise of a thick airfoil in anisotropic turbulence with $l_{a} / l_{t}<1$, especially at high frequencies. 
Synthetic turbulence methods that only enforce the transverse fluctuating velocity component under-predict the leading edge noise of thick airfoils at non-zero angle of attack. ${ }^{11,12}$ In a way, this also occurs in anisotropic turbulence with $l_{a} / l_{t}<1$, since the transverse fluctuating velocity component may become notably larger than the streamwise component and is thus the dominant component, producing the noise reduction at non-zero angle of attack.

In anisotropic turbulence with $l_{a} / l_{t}>1, \Delta \mathrm{PWL}$ spectra present an increase of about $1 \mathrm{~dB}$ at all frequencies. Although such an increase is within the level of accuracy of the current methodology, the result indicates that a moderate increase in the angle of attack of a thick airfoil has little impact on PWL spectra. In contrast, Devenport et al. ${ }^{7}$ showed an increase in SPL of about $5 \mathrm{~dB}$ for every $4^{\circ}$ increment at $800 \mathrm{~Hz}$ for a NACA 0015 airfoil in a $M_{x}=0.08$ flow with $l_{a} / l_{t}=2$, suggesting a stronger effect of anisotropy on noise. The results of the current work, which have been successfully validated by using Amiet's theory ${ }^{3}$ in Section V.A, are consistent with experimental findings. Although turbulence is considered nearly isotropic in open-jet wind tunnel experiments of leading edge noise, a certain degree of anisotropy exists. ${ }^{5,19,56}$ For instance, Moreau and Roger ${ }^{19}$ reported negligible variations in SPL spectra due to an increase of the angle of attack of a NACA 0012 airfoil, despite correcting the von Kármán and Liepmann isotropic spectra with an exponential decay function to obtain a better fit in the inertial subrange with experimental measurements. Additionally, the use of isotropic spectra in analytical models for the prediction of fan-OGV interaction noise usually offers an acceptable agreement with experiments, ${ }^{20,39}$ in which fan wakes often present $l_{a} / l_{t}>1$, suggesting that potential anisotropy effects might be negligible.

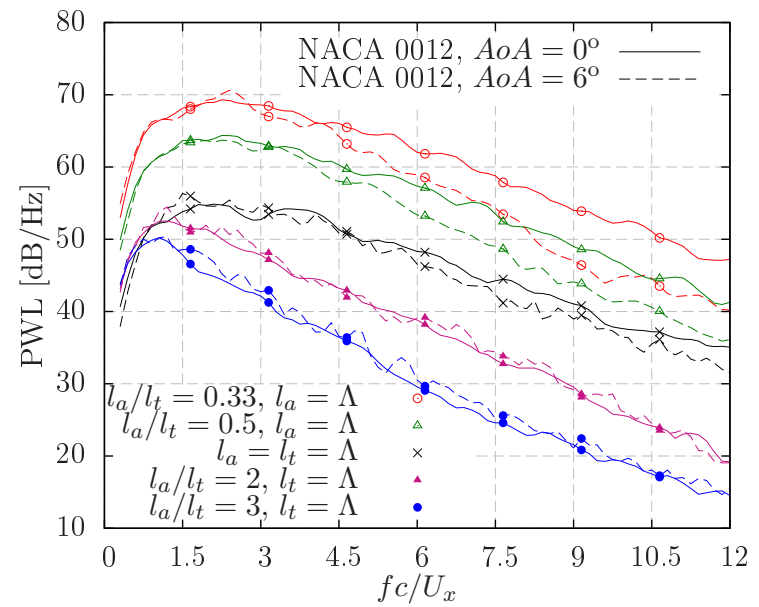

(a) PWL spectra.

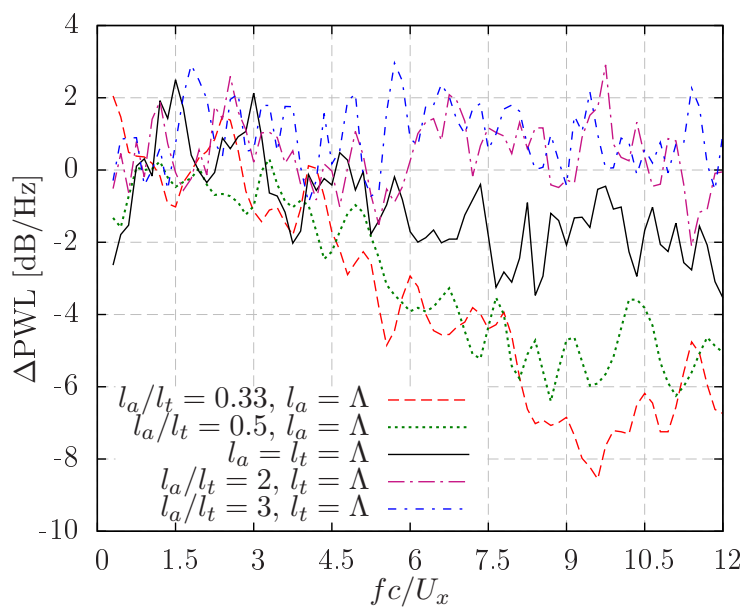

(b) $\Delta \mathrm{PWL}=\left.\mathrm{PWL}\right|_{A o A=6^{\circ}}-\left.\mathrm{PWL}\right|_{A o A=0^{\circ}}$.

Figure 12. Noise spectra from anisotropic turbulence interacting with a NACA 0012 airfoil at $M_{x}=0.3$ and $A o A=6^{\circ}$.

The discrepancy with Devenport et al.'s findings ${ }^{7}$ may be explained by the different assumptions involved in the numerical methodologies. Devenport et al. ${ }^{7}$ used a panel method that is valid for leading edge noise predictions of airfoils in incompressible flows, i.e. low Mach numbers, whereas the LEEs can deal with compressible flows. Additionally, the panel method assumed the airfoil to be acoustically compact, so that the airfoil chord is significantly smaller than the acoustic wavelength. Consequently, Devenport et al.'s noise predictions $^{7}$ were restricted to frequencies below $1000 \mathrm{~Hz}$. The validity of the results presented in the current work extends to frequencies up to $8160 \mathrm{~Hz}$ for $M_{x}=0.3\left(f c / U_{x}=12\right)$. Furthermore, the loss of compactness, which causes lobes at high frequencies in directivity plots, is correctly modeled by the LEEs.

Figure 13 shows directivity plots of NACA 0012 airfoils at chord-based Strouhal numbers and $l_{a} / l_{t}$ ratios. The leading edge noise reduction that was identified for the airfoil at $A o A=6^{\circ}$ in anisotropic turbulence with $l_{a} / l_{t}<1$ is mainly produced at downstream observer angles. For cases with anisotropic turbulence stretched in the streamwise direction, no significant variations in SPL are observed at a particular observer location due to a change in angle of attack. 


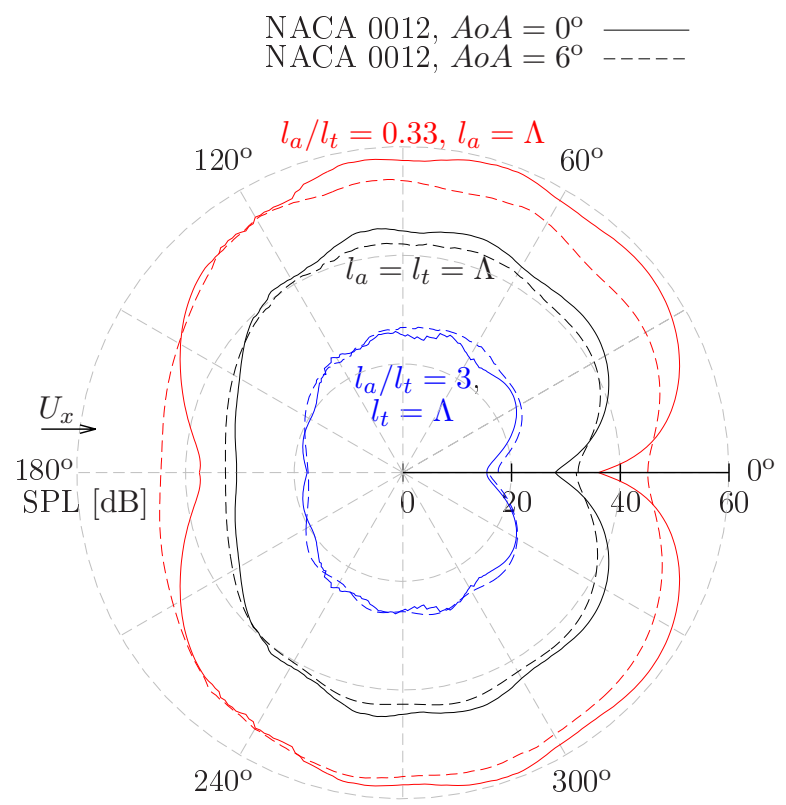

(a) $f c / U_{x}=6$.

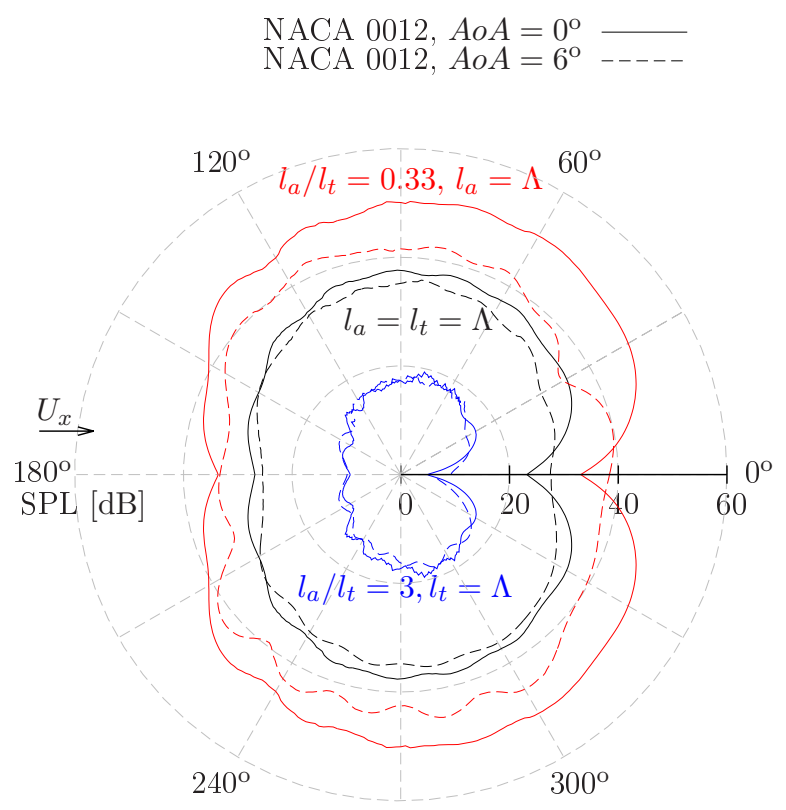

(b) $f c / U_{x}=9$.

Figure 13. Directivity plots for a NACA 0012 airfoil at $A o A=0^{\circ}$ and $A o A=6^{\circ}$ in a $M_{x}=0.3$ flow.

\section{Conclusions}

An advanced digital filter method has been presented for the generation of two-dimensional anisotropic synthetic turbulence that is suitable for leading edge noise predictions. The method realizes homogeneous anisotropic turbulence with well-defined velocity spectra through a superposition of anisotropic Gaussian spectra. To this end, a simple implementation strategy using an inlet section is applied to introduce anisotropic Gaussian eddies into the CAA domain. The method is applied to reproduce homogeneous axisymmetric turbulence with $l_{a} / l_{t}$ ratios between 0.33 and 3 , which is representative of the anisotropy in fan wakes.

Leading edge noise predictions of a number of airfoil configurations have been performed using a CAA solver to model the turbulence-airfoil interaction and a FW-H solver for the far-field noise radiation. The CAA methodology has been validated on a NACA 0001 airfoil at $A o A=0^{\circ}$ in homogeneous axisymmetric turbulence. Noise predictions show an agreement within $2 \mathrm{~dB}$ with the analytical flat plate solution. It is concluded that leading edge noise from thin airfoils mainly depends on the transverse velocity spectrum, regardless of the degree of anisotropy. The directional dependence of the length scales in anisotropic turbulence allows the energy to be redistributed over the different wavenumber components. This redistribution affects the amount of energy in the turbulent flow that contributes to leading edge noise.

CAA simulations of a NACA 0012 airfoil at $A o A=0^{\circ}$ shows that noise reduction due to airfoil thickness follows similar trends in both isotropic and moderately anisotropic turbulence. In particular, a $5 \mathrm{~dB}$ noise reduction is observed at chord-based Strouhal numbers of $f c / U_{x}=7$ in a $M_{x}=0.3$ flow. PWL $\left.\right|_{\text {flat plate }}-$ $\left.\mathrm{PWL}\right|_{\text {NACA } 0012}$ scales linearly with $f / U_{x}$, and shows a good overlap for the different $l_{a} / l_{t}$ ratios investigated at $M_{x}=0.3$. As the Mach number is increased, the onset of thickness effects on noise seems to be slightly delayed for cases with $l_{a} / l_{t}>1$. This is believed to be related to the streamwise fluctuating velocity component, which becomes increasingly important with a larger $l_{a} / l_{t}$ ratio.

An angle of attack increase of $6^{\circ}$ does not show significant changes on PWL spectra of a NACA 0012 airfoil in anisotropic turbulence with $l_{a} / l_{t}>1$. In contrast, PWL spectra decays notably faster at high frequencies when $l_{a} / l_{t}<1$ due to a noise reduction at downstream observer angles. 


\section{Acknowledgements}

The research funding for this project was partially provided by the University of Southampton and Innovate UK (Technology Strategy Board), as part of the wHole AiRcraft Multidisciplinary nOise desigN sYstem (HARMONY) program.

\section{Appendices}

\section{A. Input parameters for anisotropic Gaussian superposition}

In this appendix, representative values of $u_{0, i}, l_{x, i}$ and $l_{y, i}$ are specified for the anisotropic Gaussian superposition presented in Section IV.B.1. These values are found by fitting $N_{e}$ anisotropic Gaussian velocity spectra with the analytical expressions of homogeneous axisymmetric turbulence, assuming $k_{z}=0$. It should be noted that there is not a unique set of values for $u_{0, i}, l_{x . i}$ and $l_{y, i}$ that may be used in the anisotropic Gaussian superposition.

In all anisotropic cases investigated, $u_{a} / U_{x}=0.017$ and $u_{t}$ is not explicitly given since it disappears from Eqs. 14 and 15 with $k_{z}=0$. Nevertheless, $u_{t}$ is assumed to take a value that satisfy the constraint $2\left(u_{t} / u_{a}\right)^{2} \geq\left(l_{t} / l_{a}\right)^{2}$, for which the axisymmetric turbulence model is valid. Table A1 presents the parameters for $N_{e}=4$ anisotropic Gaussian spectra. These are used to reproduce Liepmann isotropic turbulence, which is obtained by setting $u_{r m s}=u_{a}=u_{t}$ and $\Lambda=l_{a}=l_{t}$ in the axisymmetric turbulence model, where $\Lambda=0.008 \mathrm{~m}$. This case is taken as a baseline for the study of anisotropic turbulence. Only one length scale is varied at a time in each case of study. Tables A2 and A3 present parameters to generate anisotropic turbulence with $l_{a} / l_{t}>1$, using $N_{e}=5$ anisotropic Gaussian spectra. Similarly, Tables A4 and A5 present parameters to generate anisotropic turbulence with $l_{a} / l_{t}<1$. In the energy-containing range, anisotropic Gaussian spectra usually have $l_{x, i} / l_{y, i}$ ratios close to the $l_{a} / l_{t}$ ratio of the target axisymmetric spectra.

\begin{tabular}{|c|c|c|}
\hline$l_{x, i}=l_{y, i}[\mathrm{~m}]$ & $\begin{array}{l}u_{0, i}^{2}\left[\mathrm{~m}^{2} / \mathrm{s}^{2}\right] \\
\left(M_{x}=0.3\right)\end{array}$ & $\begin{array}{l}u_{0, i}^{2}\left[\mathrm{~m}^{2} / \mathrm{s}^{2}\right] \\
\left(M_{x}=0.6\right)\end{array}$ \\
\hline $2.35 \times 10^{-2}$ & $7.38 \times 10^{-4}$ & $3.0 \times 10^{-3}$ \\
\hline $1.3 \times 10^{-2}$ & $1.77 \times 10^{-3}$ & $7.0 \times 10^{-3}$ \\
\hline $7.2 \times 10^{-3}$ & $7.1 \times 10^{-4}$ & $2.5 \times 10^{-3}$ \\
\hline $5.0 \times 10^{-3}$ & $5.0 \times 10^{-4}$ & $2.0 \times 10^{-3}$ \\
\hline
\end{tabular}

Table A1. Parameters to realize homogeneous axisymmetric turbulence with $l_{a}=l_{t}=\Lambda$.

\begin{tabular}{|c|c|c|c|}
\hline$l_{x, i}[\mathrm{~m}]$ & $l_{y, i}[\mathrm{~m}]$ & $\begin{array}{l}u_{0, i}^{2}\left[\mathrm{~m}^{2} / \mathrm{s}^{2}\right] \\
\left(M_{x}=0.3\right)\end{array}$ & $\begin{array}{l}u_{0, i}^{2}\left[\mathrm{~m}^{2} / \mathrm{s}^{2}\right] \\
\left(M_{x}=0.6\right)\end{array}$ \\
\hline $4.7 \times 10^{-2}$ & $2.35 \times 10^{-2}$ & $3.9 \times 10^{-4}$ & $1.56 \times 10^{-3}$ \\
\hline $2.6 \times 10^{-2}$ & $1.3 \times 10^{-2}$ & $9.0 \times 10^{-4}$ & $3.6 \times 10^{-3}$ \\
\hline $1.42 \times 10^{-2}$ & $7.2 \times 10^{-3}$ & $4.2 \times 10^{-4}$ & $1.68 \times 10^{-3}$ \\
\hline $8.0 \times 10^{-3}$ & $4.0 \times 10^{-3}$ & $1.8 \times 10^{-4}$ & $7.2 \times 10^{-4}$ \\
\hline $4.4 \times 10^{-3}$ & $8.0 \times 10^{-3}$ & $4.5 \times 10^{-6}$ & $1.8 \times 10^{-5}$ \\
\hline
\end{tabular}

Table A2. Parameters to realize homogeneous axisymmetric turbulence with $l_{a} / l_{t}=2$ and $l_{t}=\Lambda$.

\begin{tabular}{|c|c|c|c|}
\hline$l_{x, i}[\mathrm{~m}]$ & $l_{y, i}[\mathrm{~m}]$ & $\begin{array}{c}u_{0, i}^{2}\left[\mathrm{~m}^{2} / \mathrm{s}^{2}\right] \\
\left(M_{x}=0.3\right)\end{array}$ & $\begin{array}{l}u_{0, i}^{2}\left[\mathrm{~m}^{2} / \mathrm{s}^{2}\right] \\
\left(M_{x}=0.6\right)\end{array}$ \\
\hline $7.5 \times 10^{-2}$ & $2.5 \times 10^{-2}$ & $1.4 \times 10^{-4}$ & $6.5 \times 10^{-4}$ \\
\hline $4.7 \times 10^{-2}$ & $1.65 \times 10^{-2}$ & $4.6 \times 10^{-4}$ & $1.91 \times 10^{-3}$ \\
\hline $2.8 \times 10^{-2}$ & $9.5 \times 10^{-3}$ & $4.1 \times 10^{-4}$ & $1.45 \times 10^{-3}$ \\
\hline $1.5 \times 10^{-2}$ & $5.1 \times 10^{-3}$ & $2.2 \times 10^{-4}$ & $8.5 \times 10^{-4}$ \\
\hline $6.55 \times 10^{-3}$ & $4.0 \times 10^{-3}$ & $1.6 \times 10^{-5}$ & $6.4 \times 10^{-5}$ \\
\hline
\end{tabular}

Table A3. Parameters to realize homogeneous axisymmetric turbulence with $l_{a} / l_{t}=3$ and $l_{t}=\Lambda$. 


\begin{tabular}{|c|c|c|c|}
\hline$l_{x, i}[\mathrm{~m}]$ & $l_{y, i}[\mathrm{~m}]$ & $\begin{array}{l}u_{a, i}^{2}\left[\mathrm{~m}^{2} / \mathrm{s}^{2}\right] \\
\left(M_{x}=0.3\right)\end{array}$ & $\begin{array}{l}u_{a, i}^{2}\left[\mathrm{~m}^{2} / \mathrm{s}^{2}\right] \\
\left(M_{x}=0.6\right)\end{array}$ \\
\hline $2.1 \times 10^{-2}$ & $4.1 \times 10^{-2}$ & $4.7 \times 10^{-3}$ & $1.9 \times 10^{-2}$ \\
\hline $1.05 \times 10^{-2}$ & $2.1 \times 10^{-2}$ & $7.8 \times 10^{-3}$ & $3.0 \times 10^{-2}$ \\
\hline $4.7 \times 10^{-3}$ & $9.6 \times 10^{-3}$ & $2.5 \times 10^{-3}$ & $1.0 \times 10^{-2}$ \\
\hline $5.3 \times 10^{-3}$ & $4.9 \times 10^{-3}$ & $1.8 \times 10^{-4}$ & $7.0 \times 10^{-4}$ \\
\hline $5.0 \times 10^{-3}$ & $1.3 \times 10^{-2}$ & $1.0 \times 10^{-5}$ & $4.0 \times 10^{-5}$ \\
\hline
\end{tabular}

Table A4. Parameters to realize homogeneous axisymmetric turbulence with $l_{a} / l_{t}=0.5$ and $l_{a}=\Lambda$.

\begin{tabular}{|c|c|c|c|}
\hline$l_{x, i}[\mathrm{~m}]$ & $l_{y, i}[\mathrm{~m}]$ & $\begin{array}{c}u_{a, i}^{2}\left[\mathrm{~m}^{2} / \mathrm{s}^{2}\right] \\
\left(M_{x}=0.3\right)\end{array}$ & $\begin{array}{l}u_{a, i}^{2}\left[\mathrm{~m}^{2} / \mathrm{s}^{2}\right] \\
\left.M_{x}=0.6\right)\end{array}$ \\
\hline $2.5 \times 10^{-2}$ & $7.8 \times 10^{-2}$ & $4.0 \times 10^{-3}$ & $1.6 \times 10^{-2}$ \\
\hline $1.65 \times 10^{-2}$ & $4.7 \times 10^{-2}$ & $1.15 \times 10^{-2}$ & $4.6 \times 10^{-2}$ \\
\hline $9.5 \times 10^{-3}$ & $2.8 \times 10^{-2}$ & $1.2 \times 10^{-2}$ & $4.8 \times 10^{-2}$ \\
\hline $5.1 \times 10^{-3}$ & $1.5 \times 10^{-2}$ & $6.0 \times 10^{-3}$ & $2.4 \times 10^{-2}$ \\
\hline $4.0 \times 10^{-3}$ & $6.55 \times 10^{-3}$ & $4.8 \times 10^{-4}$ & $1.9 \times 10^{-3}$ \\
\hline
\end{tabular}

Table A5. Parameters to realize homogeneous axisymmetric turbulence with $l_{a} / l_{t}=0.33$ and $l_{a}=\Lambda$.

\section{References}

${ }^{1}$ Astley, R. J., Agarwal, A., Holland, K. R., Joseph, P. F., Self, R. H., Smith, M. G., Sugimoto, R., Tester, B. J., "Predicting and Reducing Aircraft Noise," 14th International Congress on Sound and Vibration, Australia, 2007.

${ }^{2}$ Ganz, U. W., Joppa, P. D., Patten, T. J., Scharpf, D. F., "Boeing 18-Inch Fan Rig Broadband Noise Test," NASA Contractor Report, CR-1998-208704, 1998.

${ }^{3}$ Amiet, R. K., "Acoustic Radiation from an Airfoil in a Turbulent Stream," Journal of Sound and Vibration, Vol. 41, No. 4, pp. 407-420, 1975.

${ }^{4}$ Blandeau, V. P., Joseph, P. F., Jenkins, G., Powles, C. J., "Comparison of Sound Power Radiation from Isolated Airfoils and Cascades in a Turbulent Flow," Journal of the Acoustical Society of America, Vol. 129, No. 6, pp. 3521-3530, 2011.

${ }^{5}$ Paterson, R. W., Amiet, R. K., "Acoustic Radiation and Surface Pressure Characteristics of an Airfoil due to Incident Turbulence," NASA Contractor Report, CR-2733, 1976.

${ }^{6}$ Moreau, S., Roger, M., Jurdic, V., "Effect of Angle of Attack and Airfoil Shape on Turbulence-Interaction Noise," 11th AIAA/CEAS Aeroacoustics Conference, Monterey, California, No. AIAA 2005-2973, 2005.

${ }^{7}$ Devenport, W. J., Staubs, J. K., Glegg, S. A. L., "Sound Radiation from Real Airfoils in Turbulence," Journal of Sound and Vibration, Vol. 329, pp. 3470-3483, 2010.

${ }^{8}$ Clair, V., Polacsek, C., Le Garrec, T., Reboul, G., Gruber, M., Joseph, P., "Experimental and Numerical Investigation of Turbulence-Airfoil Noise Reduction using Wavy Edges," AIAA Journal, Vol. 51, No. 11, pp. 2695-2713, 2013.

${ }^{9}$ Wohlbrandt, A. M., Gurin, S., Ewert, R., "Simultaneous Computation of Surface and Volume Sources for Fan Broadband Noise with the Random-Particle-Mesh Method," 19th AIAA/CEAS Aeroacoustics Conference, Berlin, Germany, No. AIAA 2013-2119, 2013

${ }^{10}$ Kim, D., Lee, G-S., Cheong, C., "Inflow Broadband Noise From an Isolated Symmetric Airfoil Interacting with Incident Turbulence," Journal of Fluids and Structures, Vol. 55, pp. 428-450, 2015.

${ }^{11}$ Gill, J., Zhang, X., Joseph, P., "Single Velocity-component Modeling of Leading Edge Turbulence Interaction Noise," Journal of the Acoustical Society of America, Vol. 137, No. 6, pp. 3209-3220, 2015.

${ }^{12}$ Gea-Aguilera, F., Zhang, X., Chen, X., Gill, J., Nodé-Langlois, T., "Synthetic Turbulence Methods for Leading Edge Noise Predictions," 21st AIAA/CEAS Aeroacoustics Conference, Dallas, Texas, No. AIAA 2015-2670, 2015.

${ }^{13}$ Kantola, R. A., Warren, R. E., "Reduction of Rotor-turbulence Interaction Noise in Static Fan Noise Testing," 5th AIAA Aeroacoustics Conference, Seattle, Washington, No. AIAA 79-0656, 1979.

${ }^{14}$ Mani, R., Bekofske, K., "Experimental and Theoretical Studies of Subsonic Fan Noise," NASA Contractor Report, CR-2660, 1976.

${ }^{15}$ Kerschen, E. J., Gliebe, P. R., "Noise Caused by the Interaction of a Rotor with Anisotropic Turbulence," AIAA Journal, Vol. 19, No. 6, pp. 717-723, 1981.

${ }^{16}$ Atassi, H. M., Logue, M. M., "Fan Broadband Noise in Anisotropic Turbulence," 15th AIAA/CEAS Aeroacoustics Conference, Miami, Florida, No. AIAA 2009-3148, 2009.

${ }^{17}$ Podboy, G. G., Krupar, M. J., Helland, S. M., Hughes, C. E., "Steady and Unsteady Flow Field Measurements within a NASA 22-inch Fan Model," NASA Technical Memorandum, TM-2003-212329, 2003.

${ }^{18}$ Pope, S. B., "Turbulent Flows," Cambridge University Press, 2000.

${ }^{19}$ Moreau, S., Roger, M., "Competing Broadband Noise Mechanisms in Low-Speed Axial Fans," AIAA Journal, Vol. 45, No. 1, pp. 48-57, 2007.

${ }^{20}$ Posson, H., Moreau, S., Roger, M., "Broadband Noise Prediction of Fan Outlet Guide Vane Using a Cascade Response Function," Journal of Sound and Vibration, Vol. 330, No. 25, pp. 6153-6183, 2011.

${ }^{21}$ Kraichnan, R. H., "Diffusion by a Random Velocity Field," The Physics of Fluids, Vol. 13, No. 1, pp. 22-31, 1970.

${ }^{22}$ Smirnov, A., Shi, S., Celik, I., "Random Flow Generation Technique for Large Eddy Simulations and Particle-Dynamics Modeling," Journal of Fluids Engineering, Vol. 123, No. 2, pp. 359-371, 2001.

${ }^{23}$ Huang, S. H., Li, Q. S., Wu, J. R., "A General Inflow Turbulence Generator for Large Eddy Simulations," Journal of Wind Engineering and Industrial Aerodynamics, Vol. 98, No. 10-11, pp. 600-617, 2010.

${ }^{24}$ Castro, H. G., Paz, R. R., "A Time and Space Correlated Turbulence Synthesis Method for Large Eddy Simulations," Journal of Computational Physics, Vol. 235, pp. 742-763, 2013.

${ }^{25}$ Batten,P., Goldberg, U., Chakravarthy, S., "Interfacing Statistical Turbulence Closures with Large-Eddy Simulations," AIAA Journal, Vol. 42, No. 3, pp. 485-492, 2004. 
${ }^{26}$ Billson, M., Eriksson, L.-E., Davidson, L., Jordan, P., "Modeling of Synthetic Anisotropic Turbulence and its Sound Emission," 10th AIAA/CEAS Aeroacoustics Conference, Manchester, United Kingdom, No. AIAA 2005-2857, 2004.

${ }^{27}$ Béchara, W., Bailly, C., Lafon, P., Candel, S. M., "Stochastic Approach to Noise Modeling for Free Turbulent Flows," AIAA Journal, Vol. 32, No. 3, pp. 455-463, 1994.

${ }^{28}$ Bailly, C., Juvé, D., "A Stochastic Approach to Compute Subsonic Noise using Linearized Euler's Equations," 5th AIAA/CEAS Aeroacoustics Conference, Bellevue, Washington, No. AIAA 99-1872, 1999.

${ }^{29} \mathrm{Yu}$, R., Bai, X.-S., "A Fully Divergence-Free Method for Generation of Inhomogeneous and Anisotropic Turbulence with Large Spatial Variation," Journal of Computational Physics, Vol. 256, pp. 234-253, 2014.

${ }^{30}$ Klein, M., Sadiki, A., Janicka, J., "A Digital Filter Based Generation of Inflow Data for Spatially Developing Direct Numerical or Large Eddy Simulations," Journal of Computational Physics, Vol. 186, No. 2, pp. 652-665, 2003.

${ }^{31}$ Ewert, R., "Broadband Slat Noise Prediction based on CAA and Stochastic Sound Sources from a Fast Random ParticleMesh (RPM) Method," Journal of Computers and Fluids, Vol. 37, No. 4, pp. 369-387, 2008.

${ }^{32}$ Siefert, M., Ewert, R., "Sweeping Sound Generation in Jets Realized with a Random Particle-Mesh Method," 15th AIAA/CEAS Aeroacoustics Conference, Miami, Florida, No. AIAA 2009-3369, 2009.

${ }^{33}$ Poletto, R., Craft, T., Revell, A., "A New Divergence Free Synthetic Eddy Method for the Reproduction of Inlet Flow Conditions for LES," Journal of Flow, Turbulence and Combustion, Vol. 91, No. 3, pp. 519-539, 2013.

${ }^{34}$ Sescu, A., Hixon, R., "Towards Low-Noise Synthetic Turbulent Inflow Conditions for Aeroacoustic Calculations," International Journal for Numerical Methods in Fluids, Vol. 73, No. 12, pp. 1001-1010, 2013.

${ }^{35}$ Olsen, W., Wagner, J., "Effect of Thickness on Airfoil Surface Noise," AIAA Journal, Vol. 20, No. 3, pp. 437-439, 1982.

${ }^{36}$ Hall, A. M., Atassi, O. V., Gilson, J., Reba, R., Shannon, D., "Effect of Leading-edge Thickness on High-Speed AirfoilTurbulence Interaction Noise," 17th AIAA/CEAS Aeroacoustics Conference, Portland, Oregon, No. AIAA 2011-2861, 2011.

${ }^{37}$ Glegg, S. A. L., Devenport, W. J., "Panel Methods for Airfoils in Turbulent Flow," Journal of Sound and Vibration, Vol. 329, No. 18, pp. 3709-3720, 2010.

${ }^{38}$ Chandrasekhar, S., "The Theory of Axisymmetric Turbulence," Philosophical Transactions of the Royal Society of London, Series A, Mathematical and Physical Sciences, Vol. 242, No. 855, pp. 557-577, 1950.

${ }^{39}$ Hanson, D. B., "Theory for Broadband Noise of Rotor and Stator Cascades with Inhomogeneous Inflow Turbulence including Effects of Lean and Sweep," NASA Contractor Report, CR-2001-210762, 2001.

${ }^{40}$ Batchelor, G. K., Proudman, I., "The Effect of Rapid Distortion of a Fluid in Turbulent Motion," Quarterly Journal of Mechanics and Applied Mathematics, Vol. 7, No. 1, pp. 83-103, 1954.

${ }^{41}$ Zhang, X., Chen, X. X., and Nelson, P. A., "Computation of Spinning Modal Radiation from an Unflanged Duct," AIAA Journal, Vol. 42, No. 6, pp. 1795-1801, 2004.

${ }^{42}$ Wang, X., Hu, Z., Zhang, X., "Aeroacoustic Effects of High-lift Wing Slat Track and Cut-out System," International Journal of Aeroacoustics, Vol. 12, No. 3, pp. 283-308, 2013.

${ }^{43}$ Liu, W., Kim, J. W., Zhang, X., Angland, D., Bastien, C., "Landing Gear Noise Prediction using High-order Finite Difference Schemes," Journal of Sound and Vibration, Vol. 332, No. 14, pp. 3517-3534, 2013.

${ }^{44}$ Kim, J. W., "Optimised Boundary Compact Finite Difference Schemes for Computational Aeroacoustics," Journal of Computational Physics, Vol. 225, No. 1, pp. 995-1019, 2007.

${ }^{45} \mathrm{Kim}, \mathrm{J} . \mathrm{W}$., "High-Order Compact Filters with Variable Cut-Off Wavenumber and Stable Boundary Treatment," Journal of Computers and Fluids, Vol. 39, No. 7, pp. 1168-1182, 2010.

${ }^{46} \mathrm{Hu}$, F. Q., Hussaini, M. Y., Manthey, J., "Low-Dissipation and -Dispersion Runge-Kutta Schemes for Computational Acoustics," Journal of Computational Physics, Vol. 124, No. 1, pp. 177-191, 1996.

${ }^{47}$ Gill, J., Fattah, R., Zhang, X., "Evaluation and Development of Non-Reflective Boundary Conditions for Aeroacoustic Simulations," 21st AIAA/CEAS Aeroacoustics Conference, Dallas, Texas, No. AIAA 2015-2677, 2015.

${ }^{48} \mathrm{Kim}$, J. W., Haeri, S., "An Advanced Synthetic Eddy Method for the Computation of Aerofoil-Turbulence Interaction Noise," Journal of Computational Physics, Vol. 287, pp. 1-17, 2016.

${ }^{49}$ Dieste, M., Gabard, G., "Random Particle Methods Applied to Broadband Fan Interaction Noise," Journal of Computational Physics, Vol. 231, No. 24, pp. 8133-8151, 2012.

${ }^{50}$ Taylor, G. I., "The Spectrum of Turbulence," Proceedings of the Royal Society of London, Series A, Mathematical and Physical Sciences, Vol. 164, No. 919, pp. 476-490, 1938.

${ }^{51}$ Thomson, D. J., "Spectrum Estimation and Harmonic Analysis," Proceedings of the IEEE, Vol. 70, No. 9, pp. 1055-1096, 1982.

${ }^{52}$ Liepmann, H.W., Laufer, J., Liepmann, K., "On the Spectrum of Isotropic Turbulence," NACA Technical Note, TN $2473,1951$. 1983.

${ }^{53}$ Farassat, F., Succi, G. P., "The Prediction of Helicopter Discrete Frequency Noise," Vertica, Vol. 7, No. 4, pp. 309-320,

${ }^{54}$ Gill, J., Zhang, X., Joseph, P., "Symmetric Airfoil Geometry Effects on Leading Edge Noise," Journal of the Acoustical Society of America, Vol. 134, No. 4, pp. 2669-2680, 2013.

${ }^{55}$ Paruchuri, C., Gill, J., Narayanan, S., Joseph, P., Vanderwel, C., Zhang, X., Ganapathisubramani, B., "Aerofoil Geometry Effects on Turbulence Interaction Noise," 21st AIAA/CEAS Aeroacoustics Conference, Dallas, Texas, No. AIAA 2015-2830, 2015.

${ }^{56}$ Sjögren, T., Johansson, A. V., "Measurement and Modelling of Homogeneous Axisymmetric Turbulence," Journal of Fluid Mechanics, Vol. 374, pp. 59-90, 1998. 